\title{
PETERSON-TYPE DIMENSION FORMULAS FOR GRADED LIE SUPERALGEBRAS
}

\author{
SEOK-JIN KANG ${ }^{1}$, JAE-HOON KWON AND YOUNG-TAK OH ${ }^{2}$
}

\begin{abstract}
Let $\widehat{\Gamma}$ be a free abelian group of finite rank, let $\Gamma$ be a sub-semigroup of $\widehat{\Gamma}$ satisfying certain finiteness conditions, and let $\mathfrak{L}=\bigoplus_{(\alpha, a) \in \Gamma \times \mathbb{Z}_{2}} \mathfrak{L}_{(\alpha, a)}$ be a $\left(\Gamma \times \mathbb{Z}_{2}\right)$-graded Lie superalgebra. In this paper, by applying formal differential operators and the Laplacian to the denominator identity of $\mathfrak{L}$, we derive a new recursive formula for the dimensions of homogeneous subspaces of $\mathfrak{L}$. When applied to generalized Kac-Moody superalgebras, our formula yields a generalization of Peterson's root multiplicity formula. We also obtain a Freudenthaltype weight multiplicity formula for highest weight modules over generalized Kac-Moody superalgebras.
\end{abstract}

\section{$\S 1$. Introduction}

We first recall the binomial expansion

$$
(1-t)^{n}=\sum_{k=0}^{n}(-1)^{k}\left(\begin{array}{l}
n \\
k
\end{array}\right) t^{k} .
$$

This elementary product identity can be given a Lie-theoretic interpretation as follows: Let $\mathfrak{L}=\mathbb{C} x_{1} \oplus \cdots \oplus \mathbb{C} x_{n}$ be an $n$-dimensional abelian Lie algebra with a basis $x_{1}, \cdots, x_{n}$. Since $\mathfrak{L}$ is abelian, we have $H_{k}(\mathfrak{L})=\Lambda^{k}(\mathfrak{L})$ and

$$
\operatorname{dim} H_{k}(\mathfrak{L})=\operatorname{dim} \Lambda^{k}(\mathfrak{L})=\left(\begin{array}{l}
n \\
k
\end{array}\right)
$$

Hence the binomial expansion (1.1) can be interpreted as the Euler-Poincaré principle for the abelian Lie algebra $\mathfrak{L}$.

Received December 22, 1999.

2000 Mathematics Subject Classification: 17A70, 17B01, 17B65, 17B70, 11F03 and $11 \mathrm{~F} 22$.

${ }^{1}$ This research was supported by KOSEF Grant \#98-0701-01-5-L.

${ }^{2}$ This research was supported in part by BK21 Mathematical Sciences Division, Seoul National University. 
Next, consider the Jacobi triple product identity

$$
\prod_{n=1}^{\infty}\left(1-p^{n} q^{n}\right)\left(1-p^{n-1} q^{n}\right)\left(1-p^{n} q^{n-1}\right)=\sum_{k \in \mathbb{Z}}(-1)^{k} p^{\frac{k(k-1)}{2}} q^{\frac{k(k+1)}{2}}
$$

which arises from the theory of modular forms and theta functions. In [8], V. G. Kac discovered a character formula, called the Weyl-Kac formula, for irreducible highest weight modules over symmetrizable Kac-Moody algebras with dominant integral highest weights. When applied to the 1-dimensional trivial representation, the Weyl-Kac formula yields the denominator identity, and it was shown in [8] that the Macdonald identities ([19]) are equivalent to the denominator identities for affine Kac-Moody algebras. In particular, the denominator identity for the affine Kac-Moody algebra $A_{1}^{(1)}$ is equal to the Jacobi triple product identity.

In [2], R. E. Borcherds proved the product identity

$$
p^{-1} \prod_{\substack{m>0 \\ n \in \mathbb{Z}}}\left(1-p^{m} q^{n}\right)^{c(m n)}=j(p)-j(q)
$$

for the elliptic modular function

$$
j(q)=\sum_{n=-1}^{\infty} c(n) q^{n}=q^{-1}+196884 q+21493760 q^{2}+\cdots,
$$

and showed that (1.3) is the denominator identity for the Monster Lie algebra. The Monster Lie algebra is a special case of generalized Kac-Moody algebras and it played a crucial role in Borcherds' proof of the Moonshine Conjecture ([2], [5]).

In this paper, we consider general product identities of the form

$$
\prod_{(\alpha, a) \in \Gamma \times \mathbb{Z}_{2}}\left(1-E^{(\alpha, a)}\right)^{\nu(\alpha, a)}=1+\sum_{(\beta, b) \in \Gamma \times \mathbb{Z}_{2}} \zeta(\beta, b) E^{(\beta, b)},
$$

where $\nu(\alpha, a), \zeta(\beta, b) \in \mathbb{Z}$ and $\Gamma$ is a countable (usually infinite) subsemigroup of a free abelian group $\widehat{\Gamma}$ of finite rank with a nondegenerate symmetric bilinear form. Suppose that we have a $\left(\Gamma \times \mathbb{Z}_{2}\right)$-graded Lie superalgebra $\mathfrak{L}=\bigoplus_{(\alpha, a) \in \Gamma \times \mathbb{Z}_{2}} \mathfrak{L}_{(\alpha, a)}$ such that

$$
\begin{aligned}
& \operatorname{sdim} \mathfrak{L}_{(\alpha, a)}=(-1)^{a} \operatorname{dim} \mathfrak{L}_{(\alpha, a)}=\nu(\alpha, a), \\
& \operatorname{sdim} H(\mathfrak{L})_{(\beta, b)}=(-1)^{b} \operatorname{dim} H(\mathfrak{L})_{(\beta, b)}=\zeta(\beta, b)
\end{aligned}
$$


for all $\alpha, \beta \in \Gamma, a, b \in \mathbb{Z}_{2}$. Then the product identity (1.4) can be interpreted as the Euler-Poincaré principle for the Lie superalgebra $\mathfrak{L}$ (see Section 2). We call (1.4) the denominator identity for the $\left(\Gamma \times \mathbb{Z}_{2}\right)$-graded Lie superalgebra $\mathfrak{L}=\bigoplus_{(\alpha, a) \in \Gamma \times \mathbb{Z}_{2}} \mathfrak{L}_{(\alpha, a)}$.

In Section 3, by applying formal differential operators and the Laplacian to the denominator identity of $\mathfrak{L}$, we derive a new recursive formula for the dimensions of homogeneous subspaces of $\mathfrak{L}$ (Theorem 3.3). As an immediate application, we recover some interesting recursive relations for the colored partitions $p_{r}(n)$ and the Ramanujan's tau-function $\tau(n)$ which can be found in [20]. We also obtain a set of recursive relations for the coefficients $c(n)$ of the elliptic modular function $j$ which can be used to prove the fact that the coefficients of $j$ are completely determined by the first 3 coefficients $c(1)$, $c(2)$ and $c(3)$ (cf. [17]).

For Kac-Moody algebras, it is well-known that Peterson's root multiplicity formula determines the root multiplicities recursively ([22]). In Section 4, we show that when applied to generalized Kac-Moody superalgebras, our recursive dimension formula yields a generalization of Peterson's root multiplicity formula (Theorem 4.3 and Proposition 4.4). For this reason, we call our formula the Peterson-type dimension formula for graded Lie superalgebras. Moreover, by applying the same technique that was used in deriving our Peterson-type dimension formula, we also derive a Freudenthaltype recursive weight multiplicity formula for highest weight modules over generalized Kac-Moody superalgebras (Theorem 4.5).

In the final section, we illustrate how to apply our Peterson-type root multiplicity formula and Freudenthal-type weight multiplicity formula with the examples of rank 2 generalized Kac-Moody superalgebras and their irreducible highest weight modules. We also discuss the application of our Peterson-type root multiplicity formula to Monstrous Lie superalgebras. At the end of Section 5, we present some tables of root and weight multiplicities for these algebras and modules.

Acknowledgements. We would like to express our sincere gratitude to Hee-Chul Cho, Seung-Il Kim, and Seong-Hwa Kwon for their generous help in making the tables of root and weight multiplicities. Part of this work was completed while the first author was visiting Massachusetts Institute of Technology and Yale University in the year of 1999. He is very grateful to the faculty and staff members of those institutions for their hospitality. 
We are also very grateful to Professors Myung-Hwan Kim and Ja-Kyung Koo for many helpful discussions.

\section{$\S 2$. Graded Lie superalgebras}

We recall some basic facts about Lie superalgebras. A $\mathbb{Z}_{2}$-graded vector space $\mathfrak{L}=\mathfrak{L}_{\overline{0}} \oplus \mathfrak{L}_{\overline{1}}$ is called a Lie superalgebra if there exists a bilinear map $[]:, \mathfrak{L} \times \mathfrak{L} \rightarrow \mathfrak{L}$, called the bracket, such that

$$
\begin{aligned}
& {\left[\mathfrak{L}_{a}, \mathfrak{L}_{b}\right] \subset \mathfrak{L}_{a+b},} \\
& {[x, y]=-(-1)^{a b}[y, x]} \\
& {[x,[y, z]]=[[x, y], z]+(-1)^{a b}[y,[x, z]]}
\end{aligned}
$$

for all $x \in \mathfrak{L}_{a}, y \in \mathfrak{L}_{b}, a, b \in \mathbb{Z}_{2}$. The homogeneous elements of $\mathfrak{L}_{\overline{0}}$ (resp. $\left.\mathfrak{L}_{\overline{1}}\right)$ are called even (resp. odd).

Let $\Gamma$ be a countable (usually infinite) abelian semigroup such that every element $\alpha \in \Gamma$ can be written as a sum of elements in $\Gamma$ in only finitely many ways. Consider a $\left(\Gamma \times \mathbb{Z}_{2}\right)$-graded Lie superalgebra $\mathfrak{L}=$ $\bigoplus_{(\alpha, a) \in \Gamma \times \mathbb{Z}_{2}} \mathfrak{L}_{(\alpha, a)}$ with $\operatorname{dim} \mathfrak{L}_{(\alpha, a)}<\infty$ for all $(\alpha, a) \in \Gamma \times \mathbb{Z}_{2}$. We define the character of $\mathfrak{L}$ to be

$$
\operatorname{ch} \mathfrak{L}=\sum_{(\alpha, a) \in \Gamma \times \mathbb{Z}_{2}}\left(\operatorname{dim} \mathfrak{L}_{(\alpha, a)}\right) e^{(\alpha, a)},
$$

where $e^{(\alpha, a)}$ are the basis elements of the semigroup algebra $\mathbb{C}\left[\Gamma \times \mathbb{Z}_{2}\right]$ with the multiplication given by $e^{(\alpha, a)} e^{(\beta, b)}=e^{(\alpha+\beta, a+b)}$.

On the other hand, we define the superdimension of the homogeneous subspace $\mathfrak{L}_{(\alpha, a)}$ by

$$
\operatorname{sdim} \mathfrak{L}_{(\alpha, a)}=(-1)^{a} \operatorname{dim} \mathfrak{L}_{(\alpha, a)} \quad\left(\alpha \in \Gamma, a \in \mathbb{Z}_{2}\right) .
$$

We introduce another basis elements of $\mathbb{C}\left[\Gamma \times \mathbb{Z}_{2}\right]$ by setting $E^{(\alpha, a)}=$ $(-1)^{a} e^{(\alpha, a)}$. Clearly, $E^{(\alpha, a)} E^{(\beta, b)}=E^{(\alpha+\beta, a+b)}$. We define the supercharacter of $\mathfrak{L}$ to be

$$
\operatorname{sch} \mathfrak{L}=\sum_{(\alpha, a) \in \Gamma \times \mathbb{Z}_{2}}\left(\operatorname{sdim} \mathfrak{L}_{(\alpha, a)}\right) E^{(\alpha, a)} .
$$

Note that $\operatorname{ch} \mathfrak{L}=\operatorname{sch} \mathfrak{L}$. The only difference is that, in the supercharacter, we allow the negative coefficients. Usually, they are the elements of $\mathbb{C}\left[\left[\Gamma \times \mathbb{Z}_{2}\right]\right]$, the completion of $\mathbb{C}\left[\Gamma \times \mathbb{Z}_{2}\right]$. 
The main purpose of this paper is to find an efficient recursive formula for sdim $\mathfrak{L}_{(\alpha, a)}\left(\alpha \in \Gamma, a \in \mathbb{Z}_{2}\right)$. In deriving our superdimension formula, the crucial role is played by the denominator identity for the Lie superalgebra $\mathfrak{L}=\bigoplus_{(\alpha, a) \in \Gamma \times \mathbb{Z}_{2}} \mathfrak{L}_{(\alpha, a)}$. In the following, we briefly recall how to derive the denominator identity from the Euler-Poincaré principle for Lie superalgebra homology (see [8] and [20] for more details).

Let $\mathbb{C}$ be the trivial 1-dimensional $\mathfrak{L}$-module. The homology modules $H_{k}(\mathfrak{L})=H_{k}(\mathfrak{L}, \mathbb{C})$ are determined from the following standard complex:

$$
\cdots \rightarrow C_{k}(\mathfrak{L}) \stackrel{d_{k}}{\rightarrow} C_{k-1}(\mathfrak{L}) \stackrel{d_{k-1}}{\rightarrow} \cdots \rightarrow C_{1}(\mathfrak{L}) \stackrel{d_{1}}{\rightarrow} C_{0}(\mathfrak{L}) \rightarrow 0,
$$

where the $C_{k}(\mathfrak{L})$ are defined by

$$
C_{k}(\mathfrak{L})=\bigoplus_{p+q=k} \Lambda^{p}\left(\mathfrak{L}_{\overline{0}}\right) \otimes S^{q}\left(\mathfrak{L}_{\overline{1}}\right)
$$

and the differentials $d_{k}: C_{k}(\mathfrak{L}) \rightarrow C_{k-1}(\mathfrak{L})$ are given by

$$
\begin{aligned}
& d_{k}\left(\left(x_{1} \wedge \cdots \wedge x_{p}\right) \otimes\left(y_{1} \cdots y_{q}\right)\right) \\
& =\sum_{1 \leq s<t \leq p}(-1)^{s+t}\left(\left[x_{s}, x_{t}\right] \wedge x_{1} \wedge \cdots \wedge \widehat{x_{s}} \wedge \cdots \wedge \widehat{x_{t}} \wedge \cdots \wedge x_{p}\right) \otimes\left(y_{1} \cdots y_{q}\right) \\
& \quad+\sum_{s=1}^{p} \sum_{t=1}^{q}(-1)^{s}\left(x_{1} \wedge \cdots \wedge \widehat{x_{s}} \wedge \cdots \wedge x_{p}\right) \otimes\left(\left[x_{s}, y_{t}\right] y_{1} \cdots \widehat{y_{t}} \cdots y_{q}\right) \\
& \quad-\sum_{1 \leq s<t \leq q}\left(\left[y_{s}, y_{t}\right] \wedge x_{1} \wedge \cdots \wedge x_{p}\right) \otimes\left(y_{1} \cdots \widehat{y_{s}} \cdots \widehat{y_{t}} \cdots y_{q}\right)
\end{aligned}
$$

for $k \geq 2, x_{i} \in \mathfrak{L}_{\overline{0}}, y_{j} \in \mathfrak{L}_{\overline{1}}$ and $d_{1}=0$ (cf. [4], [6]). Since the spaces $C_{k}(\mathfrak{L})$ and the homology modules $H_{k}(\mathfrak{L})$ inherit the $\left(\Gamma \times \mathbb{Z}_{2}\right)$-gradation from that of $\mathfrak{L}$, the supercharacters of $C_{k}(\mathfrak{L})$ and $H_{k}(\mathfrak{L})$ are well-defined. Hence by the Euler-Poincaré principle, we obtain

$$
\sum_{k=0}^{\infty}(-1)^{k} \operatorname{sch} C_{k}(\mathfrak{L})=\sum_{k=0}^{\infty}(-1)^{k} \operatorname{sch} H_{k}(\mathfrak{L})
$$


Let

$$
\begin{aligned}
C(\mathfrak{L}) & =\sum_{k=0}^{\infty}(-1)^{k} C_{k}(\mathfrak{L})=\mathbb{C} \ominus \mathfrak{L} \oplus C_{2}(\mathfrak{L}) \ominus \cdots, \\
\Lambda\left(\mathfrak{L}_{\overline{0}}\right) & =\sum_{k=0}^{\infty}(-1)^{k} \Lambda^{k}\left(\mathfrak{L}_{\overline{0}}\right)=\mathbb{C} \ominus \mathfrak{L}_{\overline{0}} \oplus \Lambda^{2}\left(\mathfrak{L}_{\overline{0}}\right) \ominus \cdots, \\
S\left(\mathfrak{L}_{\overline{1}}\right) & =\sum_{k=0}^{\infty}(-1)^{k} S^{k}\left(\mathfrak{L}_{\overline{1}}\right)=\mathbb{C} \ominus \mathfrak{L}_{\overline{1}} \oplus S^{2}\left(\mathfrak{L}_{\overline{1}}\right) \ominus \cdots, \\
H(\mathfrak{L}) & =\sum_{k=1}^{\infty}(-1)^{k} H_{k}(\mathfrak{L})=-H_{1}(\mathfrak{L}) \oplus H_{2}(\mathfrak{L}) \ominus H_{3}(\mathfrak{L}) \oplus \cdots,
\end{aligned}
$$

the alternating direct sum of superspaces. Then it is easy to see that

$$
C(\mathfrak{L})=\Lambda\left(\mathfrak{L}_{\overline{0}}\right) \otimes S\left(\mathfrak{L}_{\overline{1}}\right)
$$

and that

$$
\operatorname{sch} C(\mathfrak{L})=\prod_{(\alpha, a) \in \Gamma \times \mathbb{Z}_{2}}\left(1-E^{(\alpha, a)}\right)^{\operatorname{sdim} \mathfrak{L}_{(\alpha, a)}} .
$$

Therefore, we obtain the denominator identity for the $\left(\Gamma \times \mathbb{Z}_{2}\right)$ - graded Lie superalgebra $\mathfrak{L}=\bigoplus_{(\alpha, a) \in \Gamma \times \mathbb{Z}_{2}} \mathfrak{L}_{(\alpha, a)}$ :

$$
\prod_{(\alpha, a) \in \Gamma \times \mathbb{Z}_{2}}\left(1-E^{(\alpha, a)}\right)^{\operatorname{sdim} \mathfrak{L}_{(\alpha, a)}}=1+\operatorname{sch} H(\mathfrak{L}) .
$$

We often deal with the $\Gamma$-grading on $\mathfrak{L}$ defined by

$$
\mathfrak{L}_{\alpha}=\mathfrak{L}_{(\alpha, \overline{0})} \oplus \mathfrak{L}_{(\alpha, \overline{1})} \quad(\alpha \in \Gamma)
$$

In this case, by setting $\operatorname{sdim} \mathfrak{L}_{\alpha}=\operatorname{dim} \mathfrak{L}_{(\alpha, \overline{0})}-\operatorname{dim} \mathfrak{L}_{(\alpha, \overline{1})}$ and $E^{(\alpha, a)}=E^{\alpha}$, we have

$$
\operatorname{sch} \mathfrak{L}=\left(\sum_{\alpha \in \Gamma} \operatorname{sdim} \mathfrak{L}_{\alpha}\right) E^{\alpha},
$$

and (2.2) yields the denominator identity for $\Gamma$-graded Lie superalgebra $\mathfrak{L}=\bigoplus_{\alpha \in \Gamma} \mathfrak{L}_{\alpha}$ :

$$
\prod_{\alpha \in \Gamma}\left(1-E^{\alpha}\right)^{\operatorname{sdim} \mathfrak{L}_{\alpha}}=1+\operatorname{sch} H(\mathfrak{L}) .
$$

In the next section, we will derive Peterson-type recursive formulas for $\operatorname{sdim} \mathfrak{L}_{(\alpha, a)}$ and $\operatorname{sdim} \mathfrak{L}_{\alpha}\left(\alpha \in \Gamma, a \in \mathbb{Z}_{2}\right)$. The main idea is to differentiate both sides of the denominator identities (2.2) and (2.3). 


\section{$\S 3$. Peterson-type formulas}

Let $\widehat{\Gamma}$ be a free abelian group with finite rank and let $\Gamma$ be a countable (usually infinite) semi-subgroup in $\widehat{\Gamma}$ such that every element $\alpha \in \Gamma$ can be written as a sum of elements of $\Gamma$ in only finitely many ways. Let $\widehat{\Gamma}_{\mathbb{C}}=\mathbb{C} \otimes_{\mathbb{Z}} \widehat{\Gamma}$ be the complexification of $\widehat{\Gamma}$. Choose a nondegenerate symmetric bilinear form $(\mid)$ on $\widehat{\Gamma}_{\mathbb{C}}$ and fix a pair of dual bases $\left\{u_{i}\right\}$ and $\left\{u^{i}\right\}$. Define a partial ordering on $\Gamma$ by $\lambda \geq \mu$ if and only if $\lambda-\mu \in \Gamma$ or $\lambda=\mu$. We will denote by $\lambda>\mu$ if $\lambda \geq \mu$ and $\lambda \neq \mu$.

Suppose that we have a product identity of the form

$$
\prod_{(\alpha, a) \in \Gamma \times \mathbb{Z}_{2}}\left(1-E^{(\alpha, a)}\right)^{\nu(\alpha, a)}=1+\sum_{(\beta, b) \in \Gamma \times \mathbb{Z}_{2}} \zeta(\beta, b) E^{(\beta, b)},
$$

where $\nu(\alpha, a), \zeta(\beta, b) \in \mathbb{Z}$. First, we define the partial differential operators by

$$
\partial_{i}\left(E^{(\lambda, a)}\right)=\left(\lambda \mid u_{i}\right) E^{(\lambda, a)}, \quad \partial^{i}\left(E^{(\lambda, a)}\right)=\left(\lambda \mid u^{i}\right) E^{(\lambda, a)} .
$$

Fix an element $\rho \in \widehat{\Gamma}_{\mathbb{C}}$. We define the $\rho$-directional derivative by

$$
\nabla_{\rho}\left(E^{(\lambda, a)}\right)=\sum\left(\rho \mid u_{i}\right) \partial^{i}\left(E^{(\lambda, a)}\right)=(\rho \mid \lambda) E^{(\lambda, a)}
$$

and the Laplacian by

$$
\Delta\left(E^{(\lambda, a)}\right)=\sum \partial^{i} \partial_{i}\left(E^{(\lambda, a)}\right)=(\lambda \mid \lambda) E^{(\lambda, a)} .
$$

Let

$$
C=\sum_{(\gamma, a) \in \Gamma \times \mathbb{Z}_{2}} C(\gamma, a) E^{(\gamma, a)} \text { and } C^{*}=\sum_{(\gamma, a) \in \Gamma \times \mathbb{Z}_{2}} C^{*}(\gamma, a) E^{(\gamma, a)}
$$

be the formal power series whose coefficients are given by

$$
\begin{aligned}
C(\gamma, \overline{0}) & =\sum_{d \mid \gamma} \frac{1}{d} \nu\left(\frac{\gamma}{d}, \overline{0}\right)+\sum_{\substack{d \mid \gamma \\
d: \text { even }}} \frac{1}{d} \nu\left(\frac{\gamma}{d}, \overline{1}\right), \\
C(\gamma, \overline{1}) & =\sum_{\substack{d \mid \gamma \\
d: \text { odd }}} \frac{1}{d} \nu\left(\frac{\gamma}{d}, \overline{1}\right), \\
C^{*}(\gamma, a) & =(\gamma \mid \gamma) C(\gamma, a)-\sum_{(\gamma, a)=\left(\gamma^{\prime}, a^{\prime}\right)+\left(\gamma^{\prime \prime}, a^{\prime \prime}\right)}\left(\gamma^{\prime} \mid \gamma^{\prime \prime}\right) C\left(\gamma^{\prime}, a^{\prime}\right) C\left(\gamma^{\prime \prime}, a^{\prime \prime}\right) .
\end{aligned}
$$


Proposition 3.1. Let

$$
D=\prod_{(\alpha, a) \in \Gamma \times \mathbb{Z}_{2}}\left(1-E^{(\alpha, a)}\right)^{\nu(\alpha, a)}=1+\sum_{(\beta, b) \in \Gamma \times \mathbb{Z}_{2}} \zeta(\beta, b) E^{(\beta, b)} .
$$

Then the following differential equations hold:

$$
\nabla_{\rho}(D)=-\nabla_{\rho}(C) D, \quad \Delta(D)=-C^{*} D .
$$

Proof. First, note the following basic fact:

$$
(\alpha \mid \beta)=\sum_{i}\left(\alpha \mid u_{i}\right)\left(\beta \mid u^{i}\right) \text { for all } \alpha, \beta \in \widehat{\Gamma}_{\mathbb{C}}
$$

Using the formal power series $\log (1-t)=-\sum_{k=1}^{\infty} \frac{1}{k} t^{k}$, we obtain

$$
\begin{aligned}
\nabla_{\rho}(D) & =D \nabla_{\rho}(\log D) \\
& =D \nabla_{\rho}\left(-\sum_{\substack{(\alpha, a) \in \Gamma \times \mathbb{Z}_{2} \\
k \geq 1}} \frac{1}{k} \nu(\alpha, a) E^{k(\alpha, a)}\right) \\
& =-D \nabla_{\rho}(C),
\end{aligned}
$$

which yields the first equation. For the second one, observe the following differential equation

$$
\begin{aligned}
\frac{\Delta(D)}{D} & =\sum_{i} \partial_{i}\left(\frac{\partial^{i} D}{D}\right)+\sum_{i}\left(\frac{\partial_{i} D}{D}\right)\left(\frac{\partial^{i} D}{D}\right) \\
& =\Delta(\log D)+\sum_{i} \partial_{i}(\log D) \partial^{i}(\log D) .
\end{aligned}
$$

On the other hand, note that

$$
\Delta(\log D)=-\sum_{\substack{(\alpha, a) \in \Gamma \times \mathbb{Z}_{2} \\ k \geq 1}}(k \alpha \mid \alpha) \nu(\alpha, a) E^{k(\alpha, a)}
$$

and that

$$
\begin{aligned}
& \sum_{i} \partial_{i}(\log D) \partial^{i}(\log D) \\
= & \sum_{i}\left\{-\sum_{\substack{\left(\alpha^{\prime}, a^{\prime}\right) \in \Gamma \times \mathbb{Z}_{2} \\
p \geq 1}}\left(\alpha^{\prime} \mid u_{i}\right) \nu\left(\alpha^{\prime}, a^{\prime}\right) E^{p\left(\alpha^{\prime}, a^{\prime}\right)}\right\}
\end{aligned}
$$




$$
\begin{gathered}
\times\left\{-\sum_{\substack{\left(\alpha^{\prime \prime}, a^{\prime \prime}\right) \in \Gamma \times \mathbb{Z}_{2} \\
q \geq 1}}\left(\alpha^{\prime \prime} \mid u^{i}\right) \nu\left(\alpha^{\prime \prime}, a^{\prime \prime}\right) E^{q\left(\alpha^{\prime \prime}, a^{\prime \prime}\right)}\right\} \\
=\sum_{\substack{\left(\alpha^{\prime}, a^{\prime}\right),\left(\alpha^{\prime \prime}, a^{\prime \prime}\right) \in \Gamma \times \mathbb{Z}_{2} \\
p, q \geq 1}}\left(\alpha^{\prime} \mid \alpha^{\prime \prime}\right) \nu\left(\alpha^{\prime}, a^{\prime}\right) \nu\left(\alpha^{\prime}, a^{\prime \prime}\right) E^{p\left(\alpha^{\prime}, a^{\prime}\right)+q\left(\alpha^{\prime \prime}, a^{\prime \prime}\right)} .
\end{gathered}
$$

Combining the above identities, we have

$$
\begin{aligned}
\frac{\Delta(D)}{D}= & -\sum_{\substack{(\alpha, a) \in \Gamma \times \mathbb{Z}_{2} \\
k \geq 1}} k \nu(\alpha, a)(\alpha \mid \alpha) E^{k(\alpha, a)} \\
& +\sum_{\substack{\left(\alpha^{\prime}, a^{\prime}\right),\left(\alpha^{\prime \prime}, a^{\prime \prime}\right) \in \Gamma \times \mathbb{Z}_{2} \\
p, q \geq 1}} \nu\left(\alpha^{\prime}, a^{\prime}\right) \nu\left(\alpha^{\prime \prime}, a^{\prime \prime}\right)\left(\alpha^{\prime} \mid \alpha^{\prime \prime}\right) E^{p\left(\alpha^{\prime}, a^{\prime}\right)+q\left(\alpha^{\prime \prime}, a^{\prime \prime}\right)} \\
= & -\sum_{\substack{(\beta, b) \in \Gamma \times \mathbb{Z}_{2}\\
}}(\beta \mid \beta) C(\beta, b) E^{(\beta, b)} \\
& \sum_{\left(\beta^{\prime}, b^{\prime}\right),\left(\beta^{\prime \prime}, b^{\prime \prime}\right) \in \Gamma \times \mathbb{Z}_{2}}\left(\beta^{\prime} \mid \beta^{\prime \prime}\right) C\left(\beta^{\prime}, b^{\prime}\right) C\left(\beta^{\prime \prime}, b^{\prime \prime}\right) E^{\left(\beta^{\prime}+\beta^{\prime \prime}, b^{\prime}+b^{\prime \prime}\right)}
\end{aligned}
$$

It follows that

$$
\Delta(D)=-C^{*} D
$$

By comparing the coefficients of both sides in Proposition 3.1, we obtain the following recursive relations between $\nu(\alpha, a)$ and $\zeta(\alpha, a)\left(\alpha \in \Gamma, a \in \mathbb{Z}_{2}\right)$.

TheOrem 3.2. For $(\alpha, a) \in \Gamma \times \mathbb{Z}_{2}$, we have

$$
\begin{aligned}
& (\rho \mid \alpha) C(\alpha, a)+\sum_{\substack{\beta<\alpha \\
b \in \mathbb{Z}_{2}}}(\rho \mid \beta) C(\beta, b) \zeta(\alpha-\beta, a-b)=-(\rho \mid \alpha) \zeta(\alpha, a), \\
& C^{*}(\alpha, a)+\sum_{\substack{\beta<\alpha \\
b \in \mathbb{Z}_{2}}} C^{*}(\beta, b) \zeta(\alpha-\beta, a-b)=-(\alpha \mid \alpha) \zeta(\alpha, a) .
\end{aligned}
$$

As a direct consequence of (2.2) and (3.2), we obtain the main result of this paper: the Peterson-type recursive superdimension formulas for graded Lie superalgebras. 
THEOREM 3.3. Let $\mathfrak{L}=\bigoplus_{(\alpha, a) \in \Gamma \times \mathbb{Z}_{2}} \mathfrak{L}_{(\alpha, a)}$ be a $\left(\Gamma \times \mathbb{Z}_{2}\right)$-graded Lie superalgebra with finite dimensional homogeneous subspaces and define

$$
\begin{aligned}
C(\gamma, \overline{0})= & \sum_{d \mid \gamma} \frac{1}{d} \operatorname{sdim} \mathfrak{L}_{\left(\frac{\gamma}{d}, \overline{0}\right)}+\sum_{\substack{d \mid \gamma \\
d: \text { even }}} \frac{1}{d} \operatorname{sdim} \mathfrak{L}_{\left(\frac{\gamma}{d}, \overline{1}\right)}, \\
C(\gamma, \overline{1})= & \sum_{\substack{d \mid \gamma \\
d: \text { odd }}} \frac{1}{d} \operatorname{sdim} \mathfrak{L}_{\left(\frac{\gamma}{d}, \overline{1}\right)}, \\
C^{*}(\gamma, a)= & (\gamma \mid \gamma) C(\gamma, a)-\sum_{(\gamma, a)=\left(\gamma^{\prime}, a^{\prime}\right)+\left(\gamma^{\prime \prime}, a^{\prime \prime}\right)}\left(\gamma^{\prime} \mid \gamma^{\prime \prime}\right) C\left(\gamma^{\prime}, a^{\prime}\right) C\left(\gamma^{\prime \prime}, a^{\prime \prime}\right) .
\end{aligned}
$$

Then we have

$$
\begin{aligned}
& (\rho \mid \alpha) C(\alpha, a) \\
= & -\sum_{\substack{\beta<\alpha \\
b \in \mathbb{Z}_{2}}}(\rho \mid \beta) C(\beta, b) \operatorname{sdim} H(\mathfrak{L})_{(\alpha-\beta, a-b)}-(\rho \mid \alpha) \operatorname{sdim} H(\mathfrak{L})_{(\alpha, a)}, \\
(3.6) \quad & C^{*}(\alpha, a) \\
= & -\sum_{\substack{\beta<\alpha \\
b \in \mathbb{Z}_{2}}} C^{*}(\beta, b) \operatorname{sdim} H(\mathfrak{L})_{(\alpha-\beta, a-b)}-(\alpha \mid \alpha) \operatorname{sdim} H(\mathfrak{L})_{(\alpha, a)} .
\end{aligned}
$$

We return to the product identity (3.1). By setting $\nu(\alpha)=\nu(\alpha, \overline{0})+$ $\nu(\alpha, \overline{1}), \zeta(\beta)=\zeta(\beta, \overline{0})+\zeta(\beta, \overline{1})$, and $E^{\alpha}=E^{(\alpha, \overline{0})}=E^{(\alpha, \overline{1})}$, the product identity (3.1) gives rise to another product identity of the form

$$
\prod_{\alpha \in \Gamma}\left(1-E^{\alpha}\right)^{\nu(\alpha)}=1+\sum_{\beta \in \Gamma} \zeta(\beta) E^{\beta} .
$$

Let

$$
C=\sum_{\gamma \in \Gamma} C(\gamma) E^{\gamma} \quad \text { and } \quad C^{*}=\sum_{\gamma \in \Gamma} C^{*}(\gamma) E^{\gamma}
$$

be the formal power series whose coefficients are given by

$$
\begin{aligned}
C(\gamma) & =C(\gamma, \overline{0})+C(\gamma, \overline{1})=\sum_{d \mid \gamma} \frac{1}{d} \nu\left(\frac{\gamma}{d}\right), \\
C^{*}(\gamma) & =(\gamma \mid \gamma) C(\gamma)-\sum_{\gamma=\gamma^{\prime}+\gamma^{\prime \prime}}\left(\gamma^{\prime} \mid \gamma^{\prime \prime}\right) C\left(\gamma^{\prime}\right) C\left(\gamma^{\prime \prime}\right),
\end{aligned}
$$


and define the partial differential operators, the $\rho$-directional derivatives, and the Laplacian by

$$
\begin{aligned}
\partial_{i}\left(E^{\lambda}\right) & =\left(\lambda \mid u_{i}\right) E^{\lambda}, \quad \partial^{i}\left(E^{\lambda}\right)=\left(\lambda \mid u^{i}\right) E^{\lambda} \\
\nabla_{\rho}\left(E^{\lambda}\right) & =\sum\left(\rho \mid u_{i}\right) \partial^{i}\left(E^{\lambda}\right)=(\rho \mid \lambda) E^{\lambda} \\
\Delta\left(E^{\lambda}\right) & =\sum \partial^{i} \partial_{i}\left(E^{\lambda}\right)=(\lambda \mid \lambda) E^{\lambda}
\end{aligned}
$$

Then we can derive the Peterson-type recursive superdimension formulas for $\Gamma$-graded Lie superalgebras.

THEOREM 3.4. (a) Let

$$
D=\prod_{\alpha \in \Gamma}\left(1-E^{\alpha}\right)^{\nu(\alpha)}=1+\sum_{\beta \in \Gamma} \zeta(\beta) E^{\beta} .
$$

Then the following differential equations hold:

$$
\nabla_{\rho}(D)=-\nabla_{\rho}(C) D, \quad \Delta(D)=-C^{*} D
$$

(b) For any $\alpha \in \Gamma$, we have

$$
\begin{aligned}
& (\rho \mid \alpha) C(\alpha)+\sum_{\beta<\alpha}(\rho \mid \beta) C(\beta) \zeta(\alpha-\beta)=-(\rho \mid \alpha) \zeta(\alpha), \\
& C^{*}(\alpha)+\sum_{\beta<\alpha} C^{*}(\beta) \zeta(\alpha-\beta)=-(\alpha \mid \alpha) \zeta(\alpha) .
\end{aligned}
$$

TheOREM 3.5. Let $\mathfrak{L}=\bigoplus_{\alpha \in \Gamma} \mathfrak{L}_{\alpha}$ be a $\Gamma$-graded Lie superalgebra with finite dimensional homogeneous subspaces and set

$$
\begin{aligned}
C(\gamma) & =\sum_{d \mid \gamma} \frac{1}{d} \operatorname{sdim} \mathfrak{L}_{\frac{\gamma}{d}}, \\
C^{*}(\gamma) & =(\gamma \mid \gamma) C(\gamma)-\sum_{\gamma=\gamma^{\prime}+\gamma^{\prime \prime}}\left(\gamma^{\prime} \mid \gamma^{\prime \prime}\right) C\left(\gamma^{\prime}\right) C\left(\gamma^{\prime \prime}\right) .
\end{aligned}
$$

Then we have

$(3.12)(\rho \mid \alpha) C(\alpha)=-\sum_{\beta<\alpha}(\rho \mid \beta) C(\beta) \operatorname{sdim} H(\mathfrak{L})_{\alpha-\beta}-(\rho \mid \alpha) \operatorname{sdim} H(\mathfrak{L})_{\alpha}$, 


$$
C^{*}(\alpha)=-\sum_{\beta<\alpha} C^{*}(\beta) \operatorname{sdim} H(\mathfrak{L})_{\alpha-\beta}-(\alpha \mid \alpha) \operatorname{sdim} H(\mathfrak{L})_{\alpha}
$$

Remark. We can determine the exponents $\nu(\alpha, a)$ (resp. $\nu(\alpha))$ in the left-hand side of $(3.1)$ (resp. (3.7)) recursively from the coefficients $\zeta(\beta, b)$ (resp. $\zeta(\beta))$ in the right-hand side of (3.1) (resp. (3.7)), and vice versa. In other words, we can determine the superdimensions of homogeneous subspaces of graded Lie superalgebras recursively from the superdimensions of homogeneous subspaces of its homology modules, and vice versa.

ExAmPLE 3.6. (a) Suppose that $\operatorname{rank} \widehat{\Gamma}=1$. Take $\widehat{\Gamma}=\mathbb{Z}, \Gamma=\mathbb{Z}_{>0}$, and consider the product identity of the form

$$
\prod_{n=1}^{\infty}\left(1-q^{n}\right)^{\nu(n)}=\sum_{n=0}^{\infty} \zeta(n) q^{n}
$$

where $\nu(n), \zeta(n) \in \mathbb{Z}$ and $\zeta(0)=1$. Let $V=\bigoplus_{n=1}^{\infty} V_{n}$ be a $\mathbb{Z}_{>0 \text {-graded }}$ superspace with $\operatorname{sdim} V_{n}=-\zeta(n)$ for all $n \in \mathbb{Z}_{>0}$, and let $\mathfrak{L}=\bigoplus_{n=1}^{\infty} \mathfrak{L}_{n}$ be the free Lie superalgebra generated by $V$. Then the identity (3.14) can be interpreted as the denominator identity for the free Lie superalgebra $\mathfrak{L}$ and hence we have $\operatorname{sdim} \mathfrak{L}_{n}=\nu(n)\left(n \in \mathbb{Z}_{>0}\right)$ (see [13]).

Take $\rho=1$ and let $(\mid)$ be the multiplication in $\mathbb{C}$. Then by Theorem $3.4(\mathrm{~b})$, we obtain the following recursive relations between $\nu(n)$ and $\zeta(n)$ :

$$
\sum_{k=1}^{n}\left(\sum_{d \mid k} d \nu(d)\right) \zeta(n-k)=-n \zeta(n) .
$$

In particular, we obtain

$$
\operatorname{sdim} \mathfrak{L}_{n}=-\zeta(n)-\frac{1}{n} \sum_{k=1}^{n-1}\left(\sum_{d \mid k} d \operatorname{sdim} \mathfrak{L}_{d}\right) \zeta(n-k)-\sum_{\substack{d \mid n \\ d>1}} \frac{d}{n} \operatorname{sdim} \mathfrak{L}_{d} .
$$

(b) Let $r$ be a positive integer and set $\nu(n)=-r$ for all $n \in \mathbb{Z}_{>0}$. Then the product identity (3.14) yields the generating function for the $r$-colored partitions of positive integers:

$$
\prod_{n=1}^{\infty}\left(1-q^{n}\right)^{-r}=\sum_{n=0}^{\infty} p_{r}(n) q^{n}
$$


Hence the relation (3.15) gives the following recursive formula for $r$-colored partitions:

$$
p_{r}(n)=\frac{r}{n} \sum_{k=1}^{n} \sigma_{1}(k) p_{r}(n-k)
$$

where $\sigma_{1}(k)$ denotes the sum of all divisors of $k$.

(c) If we take $\nu(n)=24$ for all $n \in \mathbb{Z}_{>0}$, the product identity (3.14) is the definition of Ramanujan's tau-function:

$$
\prod_{n=1}^{\infty}\left(1-q^{n}\right)^{24}=\sum_{n=0}^{\infty} \tau(n+1) q^{n}
$$

Then the relation (3.15) yields the following recursive formula for the values of $\tau(n)$ :

$$
\tau(n+1)=-\frac{24}{n} \sum_{k=1}^{n} \sigma_{1}(k) \tau(n+1-k) .
$$

Remark. The recursive formulas (3.16) and (3.17) can be derived from the equation (2.10) in [20]. Our method can be regarded as a generalization of Macdonald's method to several variables.

ExAmPle 3.7. (a) Suppose that $\operatorname{rank} \widehat{\Gamma}=2$. Take $\widehat{\Gamma}=\mathbb{Z} \times \mathbb{Z}, \Gamma=$ $\mathbb{Z}_{>0} \times \mathbb{Z}_{>0}$ and consider the product identity of the form

$$
\prod_{m, n=1}^{\infty}\left(1-p^{m} q^{n}\right)^{\nu(m, n)}=1+\sum_{m, n=1}^{\infty} \zeta(m, n) p^{m} q^{n}
$$

where $\nu(m, n), \zeta(m, n) \in \mathbb{Z}$. Let $V=\bigoplus_{m, n=1}^{\infty} V_{(m, n)}$ be a $\left(\mathbb{Z}_{>0} \times \mathbb{Z}_{>0}\right)$ graded superspace with $\operatorname{sdim} V_{(m, n)}=-\zeta(m, n)$ for all $m, n \in \mathbb{Z}_{>0}$, and let $\mathfrak{L}=\bigoplus_{m, n=1}^{\infty} \mathfrak{L}_{(m, n)}$ be the free Lie superalgebra generated by $V$. Then the identity (3.18) can be interpreted as the denominator identity for the free Lie superalgebra $\mathfrak{L}$ and hence we have $\operatorname{sdim} \mathfrak{L}_{(m, n)}=\nu(m, n)$ for all $m, n \in \mathbb{Z}_{>0}($ see $[13])$.

Let $\rho=(1,0)$ and let $(\mid)$ denote the standard inner product on $\mathbb{C}^{2}$. Then by Theorem 3.4(b), we obtain the following recursive relations between $\nu(m, n)$ and $\zeta(m, n)$ :

$$
m \sum_{d \mid(m, n)} \frac{1}{d} \nu\left(\frac{m}{d}, \frac{n}{d}\right)
$$




$$
=-m \zeta(m, n)-\sum_{(m, n)=(k, l)+(s, t)} k\left(\sum_{d \mid(k, l)} \frac{1}{d} \nu\left(\frac{k}{d}, \frac{l}{d}\right)\right) \zeta(s, t) .
$$

Equivalently, we have

$$
\begin{aligned}
\nu(m, n)=-\zeta(m, n) & -\sum_{\substack{d>1 \\
d \mid(m, n)}} \frac{1}{d} \nu\left(\frac{m}{d}, \frac{n}{d}\right) \\
& -\frac{1}{m} \sum_{(m, n)=(k, l)+(s, t)} k\left(\sum_{d \mid(k, l)} \frac{1}{d} \nu\left(\frac{k}{d}, \frac{l}{d}\right)\right) \zeta(s, t) .
\end{aligned}
$$

It is worthwhile to note that the various choices of $\rho$ and bilinear forms give many different relations. For example, if we take $\rho=(0,1)$, then we get

$$
\begin{aligned}
n \sum_{d \mid(m, n)} & \frac{1}{d} \nu\left(\frac{m}{d}, \frac{n}{d}\right)=-n \zeta(m, n) \\
& \quad-\sum_{(m, n)=(k, l)+(s, t)} l\left(\sum_{d \mid(k, l)} \frac{1}{d} \nu\left(\frac{k}{d}, \frac{l}{d}\right)\right) \zeta(s, t) .
\end{aligned}
$$

(b) Recall the elliptic modular function $j$ defined by

(3.22) $j(q)-744=q^{-1}+196884 q+21473760 q^{2}+\cdots=\sum_{n=-1}^{\infty} c(n) q^{n}$

In [2], Borcherds proved the product identity

$$
p^{-1} \prod_{\substack{m>0 \\ n \in \mathbb{Z}}}\left(1-p^{m} q^{n}\right)^{c(m n)}=j(p)-j(q),
$$

which is equivalent to

$$
\prod_{m, n=1}^{\infty}\left(1-p^{m} q^{n}\right)^{c(m n)}=1-\sum_{m, n=1}^{\infty} c(m+n-1) p^{m} q^{n}
$$

This is the denominator identity for the (negative part of) Monster Lie algebra which played a crucial role in Borcherds' proof of the Moonshine Conjecture ([2], [5]). 
By the relation (3.20), we obtain a recursive relation for the coefficients $c(n)$ of the elliptic modular function $j$ :

$$
\begin{aligned}
c(m n)=c(m & +n-1)-\sum_{\substack{1<d \\
d \mid(m, n)}} \frac{1}{d} c\left(\frac{m n}{d^{2}}\right) \\
& +\frac{1}{m} \sum_{(k, l)+(s, t)=(m, n)} k\left(\sum_{d \mid(k, l)} \frac{1}{d} c\left(\frac{k l}{d^{2}}\right)\right) c(s+t-1) .
\end{aligned}
$$

Applying the recursive relation (3.23) to the pairs $(2, n)$ and $(3, n)$, we have

$$
c(2 n)=c(n+1)-\frac{1}{2} c\left(\frac{n}{2}\right)+\frac{1}{2} \sum_{j=1}^{n-1} c(j) c(n-j)
$$

$$
\begin{aligned}
c(3 n)=c(n+ & 2)-\frac{1}{3} c\left(\frac{n}{3}\right)+\frac{1}{3} \sum_{j=1}^{n-1} c(j) c(n-j+1) \\
& +\frac{2}{3} \sum_{j=1}^{n-1}\left(c(2 j)+\frac{1}{2} c\left(\frac{j}{2}\right)\right) c(n-j),
\end{aligned}
$$

where $c(n)=0$ for non-integral values of $n$. On the other hand, by taking the pairs $(2,2 n)$ and $(4, n)$, we get

$$
\begin{aligned}
c(4 n)= & c(2 n+1)+\frac{1}{2}\left(c(n)^{2}-c(n)\right)+\sum_{j=1}^{n-1} c(j) c(2 n-j) \\
= & c(n+3)-\frac{\xi(n)}{2} c(n)-\frac{1}{4} c\left(\frac{n}{4}\right) \\
& +\frac{1}{4} \sum_{j=1}^{n-1} c(j) c(n-j+2)+\frac{1}{2} \sum_{j=1}^{n-1}\left(c(2 j)+\frac{1}{2} c\left(\frac{j}{2}\right)\right) c(n-j+1) \\
& +\frac{3}{4} \sum_{j=1}^{n-1}\left(c(3 j)+\frac{1}{3} c\left(\frac{j}{3}\right)\right) c(n-j)
\end{aligned}
$$

where $\xi(n)=1$ if $n$ is even and $\xi(n)=0$ if $n$ is odd. 
Combining these relations, we obtain

$$
\begin{aligned}
c(2 n+1)=- & \frac{1}{2}\left(c(n)^{2}-c(n)\right)-\sum_{j=1}^{n-1} c(j) c(2 n-j)+c(n+3) \\
& -\frac{\xi(n)}{2} c(n)-\frac{1}{4} c\left(\frac{n}{4}\right)+\frac{1}{4} \sum_{j=1}^{n-1} c(j) c(n-j+2) \\
& +\frac{1}{2} \sum_{j=1}^{n-1}\left(c(2 j)+\frac{1}{2} c\left(\frac{j}{2}\right)\right) c(n-j+1) \\
& +\frac{3}{4} \sum_{j=1}^{n-1}\left(c(3 j)+\frac{1}{3} c\left(\frac{j}{3}\right)\right) c(n-j)
\end{aligned}
$$

By applying (3.24) and (3.25) repeatedly, we can reduce (3.26) to a recursive formula for $c(2 n+1)$.

Remark. It is well-known that the coefficients $c(n)$ are determined by the first 4 coefficients: $c(1), c(2), c(3)$, and $c(5)$ (see, for example, [2]). In [17], J.-K. Koo and Y.-T. Oh showed that $c(5)$ can be expressed in terms of $c(1), c(2)$, and $c(3)$. Hence the first 3 coefficients completely determine the elliptic modular function $j$. We can derive the same result using the Peterson-type recursive formula. Actually our method can be generalized to show that the coefficients of certain class of Thompson series are completely determined by the first three coefficients (cf. [16]).

\section{$\S 4$. Generalized Kac-Moody superalgebras}

In this section, we apply our superdimension formulas to generalized Kac-Moody superalgebras to derive Peterson-type root multiplicity formula and Freudenthal-type weight multiplicity formula for generalized Kac-Moody superalgebras and their highest weight modules. We first recall some of the basic structure theory and representation theory of generalized Kac-Moody superalgebras.

Let $I$ be a countable (possibly infinite) index set. A real square matrix $A=\left(a_{i j}\right)_{i, j \in I}$ is called a Borcherds-Cartan matrix if it satisfies: (i) $a_{i i}=2$ or $a_{i i} \leq 0$ for all $i \in I$, (ii) $a_{i j} \leq 0$ if $i \neq j$, and $a_{i j} \in \mathbb{Z}$ if $a_{i i}=2$, (iii) $a_{i j}=0$ implies $a_{j i}=0$. We say that an index $i$ is real if $a_{i i}=2$ and imaginary if $a_{i i} \leq 0$ and denote by $I^{\mathrm{re}}=\left\{i \in I \mid a_{i i}=2\right\}, I^{\mathrm{im}}=\left\{i \in I \mid a_{i i} \leq 0\right\}$. Let $\underline{m}=\left(m_{i} \in \mathbb{N} \mid i \in I\right)$ be a sequence of positive integers such that $m_{i}=1$ 
for all $i \in I^{\text {re }}$. We call $\underline{m}$ the charge of $A$. In this paper, we assume that the Borcherds-Cartan matrix $A$ is symmetrizable, i.e., there is a diagonal matrix $D=\operatorname{diag}\left(s_{i} \mid i \in I\right)$ with $s_{i}>0(i \in I)$ such that $D A$ is symmetric.

Let $I^{\text {odd }}$ be a subset of $I$ and set $I^{\text {even }}=I \backslash I^{\text {odd }}$. We call $i \in I$ an even index (resp. odd index) if $i \in I^{\text {even }}$ (resp. $i \in I^{\text {odd }}$ ). The Borcherds-Cartan matrix $A$ is said to be colored by $I^{\text {odd }}$ if $a_{i j} \in 2 \mathbb{Z}_{\leq 0}$ for all $j \in I$ whenever $i \in I^{\text {re }} \cap I^{\text {odd }}$.

Let $\mathfrak{h}=\left(\bigoplus_{i \in I} \mathbb{C} h_{i}\right) \oplus\left(\bigoplus_{i \in I} \mathbb{C} d_{i}\right)$ be a complex vector space with a basis $\left\{h_{i}, d_{i} \mid i \in I\right\}$, and for each $i \in I$ define a linear functional $\alpha_{i} \in \mathfrak{h}^{*}$ by

$$
\alpha_{i}\left(h_{j}\right)=a_{j i}, \quad \alpha_{i}\left(d_{j}\right)=\delta_{i j} \text { for all } j \in I .
$$

The free abelian group $Q=\bigoplus_{i \in I} \mathbb{Z} \alpha_{i}$ generated by $\alpha_{i}$ 's $(i \in I)$ is called the root lattice associated with $A$. Let $\Pi=\left\{\alpha_{i} \mid i \in I\right\}$ and $B$ be a basis of $\mathfrak{h}^{*}$ extending $\Pi$. Set $B^{\prime}=B \backslash \Pi$. Since $A$ is assumed to be symmetrizable, there is a symmetric bilinear form $(\mid)$ on $\mathfrak{h}^{*}$ defined by

$$
\begin{aligned}
& \left(\alpha_{i} \mid \alpha_{j}\right)=s_{i} a_{i j} \text { for } i, j \in I, \\
& \left(\lambda \mid \alpha_{i}\right)=\lambda\left(s_{i} h_{i}\right) \text { for } \lambda \in B^{\prime}, \\
& (\lambda \mid \mu)=0 \text { for } \lambda, \mu \in B^{\prime} .
\end{aligned}
$$

We can also define a nondegenerate symmetric bilinear form on $\mathfrak{h}$ by

$$
\left(h_{i} \mid h\right)=\frac{1}{s_{i}} \alpha_{i}(h) \text { and }\left(d_{i} \mid d_{j}\right)=0
$$

for all $h \in \mathfrak{h}, i, j \in I$ (cf. [7],[11]).

Let $Q^{+}=\sum_{i \in I} \mathbb{Z}_{\geq 0} \alpha_{i}$ and $Q^{-}=-Q^{+}$. We define a group homomorphism deg : $Q \rightarrow \mathbb{Z}_{2}$ by

$$
\operatorname{deg} \alpha_{i}= \begin{cases}\overline{0} & \text { if } i \in I^{\text {even }} \\ \overline{1} & \text { if } i \in I^{\text {odd }}\end{cases}
$$

An element $\alpha \in Q$ is said to be even (resp. odd) if $\operatorname{deg} \alpha=\overline{0}$ (resp. $\operatorname{deg} \alpha=$ $\overline{1})$.

The generalized Kac-Moody superalgebra $\mathfrak{g}=\mathfrak{g}\left(A, \underline{m}, I^{\text {odd }}\right)$ associated with a symmetrizable Borcherds-Cartan matrix $A=\left(a_{i j}\right)_{i, j \in I}$ of charge $\underline{m}=\left(m_{i} \mid i \in I\right)$ colored by $I^{\text {odd }}$ is the Lie superalgebra generated by the 
elements $h_{i}, d_{i}(i \in I), e_{i k}, f_{i k}\left(i \in I, k=1,2, \cdots, m_{i}\right)$ with the following defining relations:

$$
\begin{aligned}
& {\left[h_{i}, h_{j}\right]=\left[h_{i}, d_{j}\right]=\left[d_{i}, d_{j}\right]=0,} \\
& {\left[h_{i}, e_{j l}\right]=a_{i j} e_{j l}, \quad\left[h_{i}, f_{j l}\right]=-a_{i j} f_{j l},} \\
& {\left[d_{i}, e_{j l}\right]=\delta_{i j} e_{j l}, \quad\left[d_{i}, f_{j l}\right]=-\delta_{i j} f_{j l},} \\
& {\left[e_{i k}, f_{j l}\right]=\delta_{i j} \delta_{k l} h_{i},} \\
& \left(a d e_{i k}\right)^{1-a_{i j}}\left(e_{j l}\right)=\left(a d f_{i k}\right)^{1-a_{i j}}\left(f_{j l}\right)=0 \quad \text { if } a_{i i}=2 \text { and } i \neq j, \\
& {\left[e_{i k}, e_{j l}\right]=\left[f_{i k}, f_{j l}\right]=0 \quad \text { if } a_{i j}=0,} \\
& \operatorname{deg} h_{i}=\operatorname{deg} d_{i}=\overline{0}, \\
& \operatorname{deg} e_{i}=\operatorname{deg} f_{i}=\overline{0} \quad \text { if } i \in I^{\text {even },} \\
& \operatorname{deg} e_{i}=\operatorname{deg} f_{i}=\overline{1} \quad \text { if } i \in I^{\text {odd }},
\end{aligned}
$$

for $i, j \in I, k=1, \cdots, m_{i}, l=1, \cdots, m_{j}$.

The abelian subalgebra $\mathfrak{h}=\left(\bigoplus_{i \in I} \mathbb{C} h_{i}\right) \bigoplus\left(\bigoplus_{i \in I} \mathbb{C} d_{i}\right)$ is called the Cartan subalgebra of $\mathfrak{g}$, and the linear functionals $\alpha_{i} \in \mathfrak{h}^{*}(i \in I)$ defined by (4.1) are called the simple roots of $\mathfrak{g}$. For each $i \in I^{\text {re }}$, let $r_{i} \in G L\left(\mathfrak{h}^{*}\right)$ be the simple reflection on $\mathfrak{h}^{*}$ defined by

$$
r_{i}(\lambda)=\lambda-\lambda\left(h_{i}\right) \alpha_{i} \text { for } \lambda \in \mathfrak{h}^{*}
$$

The subgroup $W$ of $G L\left(\mathfrak{h}^{*}\right)$ generated by the $r_{i}$ 's $\left(i \in I^{\text {re }}\right)$ is called the Weyl group of $\mathfrak{g}$.

The generalized Kac-Moody superalgebra $\mathfrak{g}=\mathfrak{g}\left(A, \underline{m}, I^{\text {odd }}\right)$ has the root space decomposition $\mathfrak{g}=\bigoplus_{\alpha \in Q} \mathfrak{g}_{\alpha}$, where

$$
\mathfrak{g}_{\alpha}=\{x \in \mathfrak{g} \mid[h, x]=\alpha(h) x \text { for all } h \in \mathfrak{h}\} .
$$

If $\mathfrak{g}_{\alpha} \neq 0$, then $\alpha$ is called a root of $\mathfrak{g}$ and $\mathfrak{g}_{\alpha}$ is called the root space of $\mathfrak{g}$ attached to $\alpha$. We say that a root $\alpha$ is real if $(\alpha \mid \alpha)>0$ and imaginary if $(\alpha \mid \alpha) \leq 0$. A root $\alpha>0$ (resp. $\alpha<0$ ) is called positive (resp. negative). One can show that all the roots are either positive or negative. We denote by $\Phi, \Phi^{+}$and $\Phi^{-}$the set of all roots, positive roots and negative roots, respectively. We also denote by $\Phi_{\overline{0}}$ (resp. $\Phi_{\overline{1}}$ ) the set of all even (resp. 
odd) roots of $\mathfrak{g}$. Define the subalgebras $\mathfrak{g}^{ \pm}=\bigoplus_{\alpha \in \Phi^{ \pm}} \mathfrak{g}_{\alpha}$. Then we have the triangular decomposition:

$$
\mathfrak{g}=\mathfrak{g}^{-} \oplus \mathfrak{h} \oplus \mathfrak{g}^{+} .
$$

For each $\alpha \in Q$, we define the superdimension of $\mathfrak{g}_{\alpha}$ to be

$$
\operatorname{sdim} \mathfrak{g}_{\alpha}=(-1)^{\operatorname{deg} \alpha} \operatorname{dim} \mathfrak{g}_{\alpha}
$$

A $\mathfrak{g}$-module $V$ is called $\mathfrak{h}$-diagonalizable if it admits a weight space decomposition $V=\bigoplus_{\mu \in \mathfrak{h}^{*}} V_{\mu}$, where

$$
V_{\mu}=\{v \in V \mid h \cdot v=\mu(h) v \text { for all } h \in \mathfrak{h}\} .
$$

If $V_{\mu} \neq 0$, then $\mu$ is called a weight of $V$ and $V_{\mu}$ is called the $\mu$-weight space. We denote by $P(V)$ the set of all weights of $V$.

For $\lambda, \nu \in \mathfrak{h}^{*}$, we define $\lambda \geq \mu$ if $\lambda-\mu \in Q^{+}$. We denote by $\mathcal{O}$ the category of $\mathfrak{h}$-diagonalizable $\mathfrak{g}$-modules with finite dimensional weight spaces such that there exist a finite number of linear functionals $\lambda_{1}, \cdots, \lambda_{s}$ satisfying $P(V) \subset \cup_{i=1}^{s} D\left(\lambda_{i}\right)$, where $D(\lambda)=\left\{\mu \in \mathfrak{h}^{*} \mid \mu \leq \lambda\right\}$. The morphisms in $\mathcal{O}$ are $\mathfrak{g}$-module homomorphisms.

An $\mathfrak{h}$-diagonalizable $\mathfrak{g}$-module $V$ is called a highest weight module with highest weight $\lambda \in \mathfrak{h}^{*}$ if there is a nonzero vector $v_{\lambda} \in V$ such that (i) $e_{i k} \cdot v_{\lambda}=0$ for all $i \in I, k=1, \cdots, m_{i}$, (ii) $h \cdot v_{\lambda}=\lambda(h) v_{\lambda}$ for all $h \in \mathfrak{h}$, (iii) $V=U(\mathfrak{g}) \cdot v_{\lambda}$, where $U(\mathfrak{g})$ is the universal enveloping superalgebra of $\mathfrak{g}$. The vector $v_{\lambda}$ is called a highest weight vector. For a highest weight module $V$ with highest weight $\lambda$, we have (i) $V=U\left(\mathfrak{g}^{-}\right) \cdot v_{\lambda}$, (ii) $V=\bigoplus_{\mu \leq \lambda} V_{\mu}$, $V_{\lambda}=\mathbb{C} v_{\lambda}$, and (iii) $\operatorname{dim} V_{\mu}<\infty$ for all $\mu \leq \lambda$. Clearly, $V$ is in category $\mathcal{O}$. A $\mathfrak{g}$-module $M(\lambda)$ with highest weight $\lambda$ is called a Verma module if every $\mathfrak{g}$-module with highest weight $\lambda$ is a quotient of $M(\lambda)$. The Verma module $M(\lambda)$ contains a unique maximal submodule $J(\lambda)$. Therefore the quotient $V(\lambda)=M(\lambda) / J(\lambda)$ is irreducible.

Take a linear functional $\rho \in \mathfrak{h}^{*}$ satisfying $\rho\left(h_{i}\right)=\frac{1}{2} a_{i i}$ for all $i \in I$. Such a linear functional is called a Weyl vector of $\mathfrak{g}$. Note that $\left(\rho \mid \alpha_{i}\right)=$ $\rho\left(s_{i} h_{i}\right)=\frac{1}{2}\left(\alpha_{i} \mid \alpha_{i}\right)(i \in I)$.

Let $P^{+}$be the set of all linear functionals $\lambda \in \mathfrak{h}^{*}$ satisfying

$$
\begin{cases}\lambda\left(h_{i}\right) \in \mathbb{Z}_{\geq 0} & \text { for all } i \in I^{\mathrm{re}} \\ \lambda\left(h_{i}\right) \in 2 \mathbb{Z}_{\geq 0} & \text { for all } i \in I^{\mathrm{re}} \cap I^{\text {odd }} \\ \lambda\left(h_{i}\right) \geq 0 & \text { for all } i \in I^{\mathrm{im}}\end{cases}
$$


The elements of $P^{+}$are called the dominant integral weights. For a dominant integral weight $\lambda \in P^{+}$, let

$$
\begin{aligned}
& \Phi^{+}(\lambda)=\left\{\beta=\sum_{i \in I^{\text {im }}} k_{i} \alpha_{i} \in Q^{+} \mid\left(\lambda \mid \alpha_{i}\right)=0 \text { for } k_{i} \geq 1\right. \\
& \left.\left(\alpha_{i} \mid \alpha_{j}\right)=0 \text { for } k_{i}, k_{j} \geq 1, i \neq j, \quad\left(\alpha_{i} \mid \alpha_{i}\right)=0 \text { for } k_{i} \geq 2\right\} .
\end{aligned}
$$

For such an element $\beta \in \Phi^{+}(\lambda)$, we denote $|\beta|=\sum_{i \in I^{\text {im }}} k_{i}$ and

$$
\varepsilon(\beta)=\prod_{i \in I^{\mathrm{im} \cap I^{\mathrm{even}}}}\left(\begin{array}{c}
m_{i} \\
k_{i}
\end{array}\right) \prod_{j \in I^{\mathrm{im} \cap I^{\text {odd }}}}\left(\begin{array}{c}
m_{j}+k_{j}-1 \\
k_{j}
\end{array}\right)
$$

For each $\mu \leq \lambda$, we define $\operatorname{deg} \mu=\left\{\begin{array}{ll}\overline{0} & \text { if } \lambda-\mu \text { is even, } \\ \overline{1} \text { if } \lambda-\mu & \text { is odd. }\end{array}\right.$ Then the supercharacters of the highest weight modules are given in the following proposition.

Proposition 4.1. ([21], [23])

(a) For any $\lambda \in \mathfrak{h}^{*}$, we have

$$
\operatorname{sch} M(\lambda)=\frac{E^{\lambda}}{\prod_{\alpha \in Q^{-}}\left(1-E^{\alpha}\right)^{\operatorname{sdim} \mathfrak{g}_{\alpha}}} .
$$

(b) For any $\lambda \in P^{+}$, we have

$$
\operatorname{sch} V(\lambda)=\frac{\sum_{\substack{w \in W \\ \beta \in \Phi^{+}(\lambda)}} \varepsilon_{\lambda}(w, \beta) E^{w(\lambda+\rho-\beta)-\rho}}{\prod_{\alpha \in \Phi^{-}}\left(1-E^{\alpha}\right)^{\operatorname{sdim} \mathfrak{g}_{\alpha}}},
$$

where $\varepsilon_{\lambda}(w, \beta)=(-1)^{l(w)+|\beta|}(-1)^{\operatorname{deg}(w(\lambda+\rho-\beta)-\rho)} \varepsilon(\beta)$.

In particular, if $\lambda=0$, we obtain the denominator identity:

$$
\prod_{\alpha \in \Phi^{-}}\left(1-E^{\alpha}\right)^{\operatorname{sdim} \mathfrak{g}_{\alpha}}=1+\sum_{\beta \in \Phi^{-}} \zeta(\beta) E^{\beta}
$$

where $\zeta(\beta)=\sum_{\substack{w \in W \\ \gamma \in \Phi^{+}(0) \\ \beta=w(\rho-\gamma)-\rho}} \varepsilon_{0}(w, \gamma)$ 
Let $J$ be a finite subset of $I^{\mathrm{re}}$, and let $\Phi_{J}=\Phi \cap\left(\sum_{j \in J} \mathbb{Z} \alpha_{j}\right), \Phi_{J}^{ \pm}=$ $\Phi^{ \pm} \cap \Phi_{J}$ and $\Phi^{ \pm}(J)=\Phi^{ \pm} \backslash \Phi_{J}^{ \pm}$. We also denote $Q_{J}=Q \cap\left(\sum_{j \in J} \mathbb{Z} \alpha_{j}\right)$, $Q_{J}^{ \pm}=Q^{ \pm} \cap Q_{J}$ and $Q^{ \pm}(J)=Q^{ \pm} \backslash Q_{J}^{ \pm}$. Let $\mathfrak{g}_{0}^{(J)}=\mathfrak{h} \oplus\left(\bigoplus_{\alpha \in \Phi_{J}} \mathfrak{g}_{\alpha}\right)$, and $\mathfrak{g}_{ \pm}^{(J)}=\bigoplus_{\alpha \in \Phi^{ \pm}(J)} \mathfrak{g}_{\alpha}$. Then we have the triangular decomposition:

$$
\mathfrak{g}=\mathfrak{g}_{-}^{(J)} \oplus \mathfrak{g}_{0}^{(J)} \oplus \mathfrak{g}_{+}^{(J)},
$$

where $\mathfrak{g}_{0}^{(J)}$ is the Kac-Moody superalgebra (with an extended Cartan subalgebra) associated with the generalized Cartan matrix $A_{J}=\left(a_{i j}\right)_{i, j \in J}$ of charge $m_{J}=\left(m_{i} \mid i \in J\right)$ colored by $I_{J}^{\text {odd }}=I^{\text {odd }} \cap J$. Let $W_{J}$ be the subgroup of $W$ generated by the simple reflections $r_{j}$ with $j \in J$, and let $W(J)=\left\{w \in W \mid \Phi_{w} \subset \Phi^{+}(J)\right\}$, where $\Phi_{w}=\left\{\alpha \in \Phi^{+} \mid w^{-1} \alpha<0\right\}$. Then $W(J)$ is the set of right coset representatives of $W_{J}$ in $W$. In the following proposition, we recall the denominator identity for the Lie superalgebra $\mathfrak{g}_{-}^{(J)}$.

Proposition 4.2. ([13],[18]) Let $J \subset I^{\mathrm{re}}$ and let $\mathfrak{g}_{-}^{(J)}=\bigoplus_{\alpha \in \Phi^{-}(J)} \mathfrak{g}_{\alpha}$ be the subalgebra of the generalized Kac-Moody superalgebra $\mathfrak{g}$ defined as above. Then we have

$$
\begin{aligned}
\prod_{\alpha \in \Phi^{-}(J)}\left(1-E^{\alpha}\right)^{\operatorname{sdim} \mathfrak{g}_{\alpha}} \\
\quad=\sum_{\substack{w \in W(J) \\
\beta \in \Phi^{+}(0)}}(-1)^{l(w)+|\beta|} \varepsilon(\beta) \operatorname{sch} V_{J}(w(\rho-\beta)-\rho),
\end{aligned}
$$

where $V_{J}(\mu)$ is the irreducible highest weight module over $\mathfrak{g}_{0}^{(J)}$ with highest weight $\mu$.

From now on, we will use the notation

$$
H\left(\mathfrak{g}_{-}^{(J)}\right)=\bigoplus_{k=0}^{\infty}(-1)^{k} H_{k}\left(\mathfrak{g}_{-}^{(J)}\right),
$$

where

$$
H_{k}\left(\mathfrak{g}_{-}^{(J)}\right)=\sum_{\substack{w \in W(J) \\ \beta \in \Phi^{+}(0) \\ l(w)+|\beta|=k}} V_{J}(w(\rho-\beta)-\rho)^{\varepsilon(\beta)}
$$


Then (4.5) can be also written as

$$
\prod_{\alpha \in \Phi^{-}(J)}\left(1-E^{\alpha}\right)^{\operatorname{sdim} \mathfrak{g}_{\alpha}}=\operatorname{sch} H\left(\mathfrak{g}_{-}^{(J)}\right)
$$

The index set $I$ is assumed to be finite so that we can be guaranteed the existence of dual bases for the nondegenerate bilinear form $(\mid)$ on $\mathfrak{h}^{*}$. Later, we will explain how we can deal with the case when $I$ is infinite (see the remark after Theorem 4.8).

Let

$$
C=\sum_{\gamma \in Q^{-}(J)} C(\gamma) E^{\gamma} \text { and } \quad C^{*}=\sum_{\gamma \in Q^{-}(J)} C^{*}(\gamma) E^{\gamma}
$$

be the formal power series whose coefficients are given by

$$
\begin{aligned}
C(\gamma) & =\sum_{d \mid \gamma} \frac{1}{d} \operatorname{sdim} \mathfrak{g}_{\frac{\gamma}{d}}, \\
C^{*}(\gamma) & =(\gamma \mid \gamma) C(\gamma)-\sum_{\substack{\gamma=\gamma^{\prime}+\gamma^{\prime \prime} \\
\gamma^{\prime}, \gamma^{\prime \prime} \in Q^{-}(J)}}\left(\gamma^{\prime} \mid \gamma^{\prime \prime}\right) C\left(\gamma^{\prime}\right) C\left(\gamma^{\prime \prime}\right)
\end{aligned}
$$

Then by Theorem 3.4, we obtain the following Peterson-type recursive root multiplicity formula for generalized Kac-Moody superalgebras.

Theorem 4.3 .

(a) Let

$$
\begin{aligned}
D^{(J)} & =\prod_{\substack{\alpha \in \Phi^{-}(J)\\
}}\left(1-E^{\alpha}\right)^{\operatorname{sdim} \mathfrak{g}_{\alpha}} \\
& =\sum_{\substack{w \in W(J) \\
\beta \in \Phi^{+}(0)}}(-1)^{l(w)+|\beta|} \varepsilon(\beta) \operatorname{sch} V_{J}(w(\rho-\beta)-\rho),
\end{aligned}
$$

where $V_{J}(\mu)$ is the irreducible highest weight module over $\mathfrak{g}_{0}^{(J)}$ with highest weight $\mu$. Then for any $\mu \in \mathfrak{h}^{*}$, the following differential equations hold:

$$
\nabla_{\mu}\left(D^{(J)}\right)=-\nabla_{\mu}(C) D^{(J)}, \quad \Delta\left(D^{(J)}\right)=-C^{*} D^{(J)} .
$$


(b) For any $\mu \in \mathfrak{h}^{*}$ and any $\gamma \in Q^{-}(J)$, we have

$$
\begin{gathered}
(\mu \mid \gamma) C(\gamma)+\sum_{\substack{\gamma=\gamma^{\prime}+\gamma^{\prime \prime} \\
\gamma^{\prime}, \gamma^{\prime \prime} \in Q^{-}(J)}}\left(\mu \mid \gamma^{\prime}\right) C\left(\gamma^{\prime}\right) \zeta\left(\gamma^{\prime \prime}\right)=-(\mu \mid \gamma) \zeta(\gamma), \\
C^{*}(\gamma)+\sum_{\substack{\gamma=\gamma^{\prime}+\gamma^{\prime \prime} \\
\gamma^{\prime}, \gamma^{\prime \prime} \in Q^{-}(J)}} C^{*}\left(\gamma^{\prime}\right) \zeta\left(\gamma^{\prime \prime}\right)=-(\gamma \mid \gamma) \zeta(\gamma),
\end{gathered}
$$

where $\zeta(\gamma)=\sum_{\substack{w \in W(J) \\ \beta \in \Phi^{+}(0)}}(-1)^{l(w)+|\beta|} \varepsilon(\beta) \operatorname{sdim} V_{J}(w(\rho-\beta)-\rho)_{\gamma}$.

Take $J=\emptyset$ and let $\rho \in \mathfrak{h}^{*}$ be a Weyl vector of $\mathfrak{g}$. Then $W(J)=W$ and by definition of $\Phi^{+}(0)$ and $W$-invariance of $(\mid)$, we have

$$
\left(\Delta+2 \nabla_{\rho}\right)\left(D^{(\emptyset)}\right)=\left(\Delta+2 \nabla_{\rho}\right)\left(\sum_{\substack{w \in W \\ \beta \in \Phi^{+}(0)}}(-1)^{l(w)+|\beta|} \varepsilon(\beta) E^{w(\rho-\beta)-\rho}\right)=0
$$

Hence we get

$$
\left(\Delta+2 \nabla_{\rho}\right)\left(D^{(\emptyset)}\right)=-\left(C^{*}+2 \nabla_{\rho}(C)\right) D^{(\emptyset)}=0
$$

which implies $C^{*}+2 \nabla_{\rho}(C)=0$. Therefore we obtain Peterson's root multiplicity formula extended to generalized Kac-Moody superalgebras.

Proposition 4.4. ([17]) For any $\gamma \in Q^{-}$, we have

$$
(\gamma+2 \rho \mid \gamma) C(\gamma)=\sum_{\substack{\gamma=\gamma^{\prime}+\gamma^{\prime \prime} \\ \gamma^{\prime}, \gamma^{\prime \prime} \in Q^{-}}}\left(\gamma^{\prime} \mid \gamma^{\prime \prime}\right) C\left(\gamma^{\prime}\right) C\left(\gamma^{\prime \prime}\right)
$$

where $C(\gamma)=\sum_{d \mid \gamma} \frac{1}{d} \operatorname{sdim} \mathfrak{g}_{\frac{\gamma}{d}}$

Remark. To calculate the root multiplicities recursively, we would like to make sure that $(\gamma \mid \gamma+2 \rho) \neq 0$. If $\gamma \in W \cdot\left(-2 \alpha_{i}\right)$ for some $i \in I^{\text {re }} \cap I^{\text {odd }}$, it may occur that $(\gamma \mid \gamma+2 \rho)=0$, but we already know that sdim $\mathfrak{g}_{\gamma}=1$. If $\gamma \notin W \cdot\left(-2 \alpha_{i}\right)$, then one can show that $(\gamma \mid \gamma+2 \rho) \leq 0$ and that the equality holds if and only if $\gamma=-\alpha_{i}$ for some $i \in I$. That is, if $\gamma$ is not a simple root and $\gamma \notin W \cdot\left(-2 \alpha_{i}\right)$ for all $i \in I^{\text {re }} \cap I^{\text {odd }}$, then we have $(\gamma \mid \gamma+2 \rho)<0$. 
For the other extreme, take $J=I^{\text {re }}$. Then $W(J)=\{1\}$ and we get

$$
\zeta(\gamma)=\sum_{\beta \in \Phi^{+}(0)}(-1)^{|\beta|} \varepsilon(\beta) \operatorname{sdim} V_{J}(-\beta)_{\gamma},
$$

which is independent of the Weyl group $W$. For example, if $a_{i j} \neq 0$ for all $i, j \in I^{\mathrm{im}}$, then every element $\beta \in \Phi^{+}(0)$ has the form $\beta=0$ or $\beta=\alpha_{i}(i \in$ $\left.I^{\mathrm{im}}\right)$. Hence we get

$$
\zeta(\gamma)= \begin{cases}1 & \text { if } \gamma=0 \\ -\sum_{i \in I^{\mathrm{im}}} m_{i} \operatorname{sdim} V_{J}\left(-\alpha_{i}\right)_{\gamma} & \text { otherwise }\end{cases}
$$

Therefore, if the weight multiplicities of irreducible highest weight $\mathfrak{g}_{0}^{(J)}$ modules are explicitly known, then the Peterson-type root multiplicity formula (4.6) gives a very efficient recursive formula for the root multiplicities of $\mathfrak{g}$.

Finally, by the same technique that was used in deriving our Petersontype root multiplicity formula, we can derive a Freundenthal-type weight multiplicity formula for highest weight modules over generalized Kac-Moody superalgebras.

THEOREM 4.5. Let $V$ be a highest weight module over a generalized Kac-Moody superalgebra with highest weight $\Lambda \in \mathfrak{h}^{*}$. Then, for any $\lambda \leq \Lambda$, we have

$$
\begin{aligned}
\left(|\Lambda+\rho|^{2}-|\lambda+\rho|^{2}\right) \operatorname{sdim} V_{\lambda} \\
\quad=2 \sum_{\substack{\alpha \in \Phi^{+} \\
j \geq 1}}(\lambda+j \alpha \mid \alpha)\left(\operatorname{sdim} \mathfrak{g}_{\alpha}\right)\left(\operatorname{sdim} V_{\lambda+j \alpha}\right) \\
\quad=2 \sum_{\beta \in Q^{+}}(\lambda+\beta \mid \beta) C(\beta) \operatorname{sdim} V_{\lambda+\beta} .
\end{aligned}
$$

Proof. Recall that ([11])

$$
\operatorname{sch} V=\sum_{\substack{\lambda \leq \Lambda \\|\lambda+\rho|^{2}=|\Lambda+\rho|^{2}}} c_{\lambda} \operatorname{sch} M(\lambda), \text { where } c_{\lambda} \in \mathbb{Z}, c_{\Lambda}=1 .
$$


For convenience, set

$$
\begin{aligned}
& A=\operatorname{sch} V, \\
& B=\prod_{\alpha \in \Phi^{-}}\left(1-E^{\alpha}\right)^{\operatorname{sdim} \mathfrak{g}_{\alpha}}, \\
& D=\sum_{\substack{\lambda \leq \Lambda \\
|\lambda+\rho|^{2}=|\Lambda+\rho|^{2}}} c_{\lambda} E^{\lambda} .
\end{aligned}
$$

Then by Proposition 4.1 (a), we have $A B=D$. Applying the Laplacian and the $\rho$-directional derivative yields

$$
\begin{aligned}
(\Delta+ & \left.2 \nabla_{\rho}\right)(A B) \\
& =A\left(\Delta+2 \nabla_{\rho}\right)(B)+B\left(\Delta+2 \nabla_{\rho}\right)(A)+2 A B \sum_{i} \partial_{i}(\log A) \partial^{i}(\log B) .
\end{aligned}
$$

On the other hand, since $|\lambda+\rho|^{2}=|\Lambda+\rho|^{2}$, we get

$$
\left(\Delta+2 \nabla_{\rho}\right)(D)=(\Lambda \mid \Lambda+2 \rho) D .
$$

Recall that $\left(\Delta+2 \nabla_{\rho}\right)(B)=0$. Hence by combining these equations, we conclude

$$
\left(\Delta+2 \nabla_{\rho}\right)(A)+2 \sum_{i} \partial_{i}(A) \partial^{i}(\log B)=(\Lambda \mid \Lambda+2 \rho) A .
$$

By comparing the coefficients of $E^{\lambda}$, we obtain the desired result.

\section{Remark.}

(a) By taking a look at the formula (4.8), a natural question arises: what would happen if $|\lambda+\rho|^{2}=|\Lambda+\rho|^{2}$ ? If $\Lambda \in P^{+}$, we can prove that $|\lambda+\rho|^{2} \leq|\Lambda+\rho|^{2}$ for all weights $\lambda \leq \Lambda$ and that the equality holds if and only if $\lambda=\Lambda$.

(b) In [17], the Freudenthal-type weight multiplicity formula was derived for generalized Kac-Moody superalgebras using the Casimir operator.

(c) Our results in this section also hold when $I$ is infinite. If $\beta=$ $\sum k_{j} \alpha_{j} \in Q^{-}$, let $J=\left\{j \in I \mid k_{j} \neq 0\right\}$ and we can apply our theorems to the generalized Kac-Moody superalgebra $\mathfrak{g}\left(A_{J}, \underline{m}_{J}, I_{J}^{\text {odd }}\right)$ which corresponds to the finite index set $J$. 


\section{$\S 5 . \quad$ Examples and tables}

In this section, we illustrate how to apply our Peterson-type root multiplicity formula with the examples of rank 2 generalized Kac-Moody superalgebras and Monstrous Lie superalgebras. We also give an example of computing the weight multiplicities for irreducible highest weight modules over rank 2 generalized Kac-Moody superalgebras. At the end of this section, we present some tables of root and weight multiplicities for these algebras and modules.

ExAmPle 5.1. Let $A=(a)$ be a rank 1 Borcherds-Cartan matrix of charge $\underline{m}=(r)$ with $I^{\text {odd }}=I$, and let $\mathfrak{g}=\mathfrak{g}\left(A, \underline{m}, I^{\text {odd }}\right)$ be the associated generalized Kac-Moody superalgebra. Here, we have $a=2, a=0$, or $a<0$, and $r \in \mathbb{Z}_{>0}$. We denote by $\alpha$ the only simple root of $\mathfrak{g}$.

If $a=2$, then $\mathfrak{g}$ is the 5 -dimensional ortho-symplectic Lie superalgebra $\operatorname{osp}(1,2)$ and we have

$$
\operatorname{sdim} \mathfrak{g}_{n \alpha}= \begin{cases}-1 & \text { if } n=1 \\ 1 & \text { if } n=2 \\ 0 & \text { if } n \geq 3\end{cases}
$$

If $a=0$, then $\mathfrak{g}$ is the Heisenberg Lie superalgebra $\operatorname{sl}(1,2 r)$ and we have

$$
\operatorname{sdim} \mathfrak{g}_{n \alpha}= \begin{cases}-r & \text { if } n=1 \\ 0 & \text { if } n \geq 2\end{cases}
$$

If $a<0$, then the simple root $\alpha$ is odd and imaginary with multiplicity $r \geq 1$. We identify the root lattice with $\mathbb{Z}$ by setting $\alpha=1$ and choose $\mu=\frac{1}{a}$ so that $(\mu \mid 1)=1$.

For $n>1$, the formula (4.6) yields

$$
-n C(-n)=\sum_{k=1}^{n-1} k C(-k) \zeta(-n+k)+n \zeta(-n) .
$$

Since $\zeta(-1)=r$ and $\zeta(-n)=0$ for $n \geq 2$, we get $n C(-n)=-r(n-$ 1) $C(-n+1)$, which gives

$$
C(-n)=C(n)=\frac{1}{n}(-r)^{n}
$$


Hence we obtain

$$
\operatorname{sdim} \mathfrak{g}_{n \alpha}=\frac{1}{n}(-r)^{n}-\sum_{\substack{d>1 \\ d \mid n}} \frac{1}{d} \operatorname{sdim} \mathfrak{g}_{\frac{n}{d} \alpha} .
$$

If we apply the Möbius inversion to (5.1), we get the Witt formula for free Lie superalgebras :

$$
\operatorname{sdim} \mathfrak{g}_{n \alpha}=\frac{1}{n} \sum_{d \mid n} \mu(d)(-r)^{\frac{n}{d}}
$$

In particular, the subalgebra $\mathfrak{g}_{+}=\bigoplus_{n \geq 1} \mathfrak{g}_{n \alpha}\left(\right.$ resp. $\left.\mathfrak{g}_{-}=\bigoplus_{n \geq 1} \mathfrak{g}_{-n \alpha}\right)$ is the free Lie superalgebra generated by the subspace $\mathfrak{g}_{\alpha}\left(\right.$ resp. $\left.\mathfrak{g}_{-\alpha}\right)$.

ExAmple 5.2. Let $I=\{0,1\}$ be the index set and let $A=\left(\begin{array}{rr}2 & -a \\ -b & -c\end{array}\right)$ $\left(a, b, c \in \mathbb{Z}_{\geq 0}\right)$ be a Borcherds-Cartan matrix of charge $\underline{m}=(1, r)$. Take $I^{\text {odd }}=\{1\}$ and let $\mathfrak{g}=\mathfrak{g}\left(A, \underline{m}, I^{\text {odd }}\right)$ be the associated generalized KacMoody superalgebra. We identify $\alpha=m \alpha_{0}+n \alpha_{1} \in Q$ with $(m, n) \in \mathbb{Z} \times \mathbb{Z}$ and choose $\mu \in \mathfrak{h}^{*}$ such that $(\mu \mid(m, n))=m$. We would like to apply our Peterson-type root multiplicity formula (4.6) with $J=\{0\}$. In this case, the subalgebra $\mathfrak{g}_{0}^{(J)}$ is isomorphic to $\operatorname{sl}(2, \mathbb{C})+\mathfrak{h}$.

(a) If $a, b, c>0$, then

$$
\begin{aligned}
& H_{0}\left(\mathfrak{g}_{-}^{(J)}\right)=\mathbb{C} \\
& H_{1}\left(\mathfrak{g}_{-}^{(J)}\right)=V_{J}\left(-\alpha_{1}\right)^{\oplus r} \\
& H_{k}\left(\mathfrak{g}_{-}^{(J)}\right)=0 \text { for } k \geq 2
\end{aligned}
$$

where $V_{J}\left(-\alpha_{1}\right)^{\oplus r}$ is the $r$-copies of $(a+1)$-dimensional irreducible $s l(2, \mathbb{C})$ module, and we have

$$
\zeta(-m,-n)=\operatorname{sdim} H\left(\mathfrak{g}_{-}^{(J)}\right)_{(-m,-n)}= \begin{cases}1 & \text { if }(m, n)=(0,0) \\ r & \text { if } 0 \leq m \leq a, n=1 \\ 0 & \text { otherwise }\end{cases}
$$

Therefore we obtain

$$
\operatorname{sdim} \mathfrak{g}_{(m, n)}=C(m, n)-\sum_{\substack{d>1 \\ d \mid(m, n)}} \frac{1}{d} \operatorname{sdim} \mathfrak{g}_{\left(\frac{m}{d}, \frac{n}{d}\right)}
$$


where $C(m, n)$ are determined recursively by

$$
C(m, n)= \begin{cases}-r & \text { if } 1 \leq m \leq a, n=1, \\ -\frac{r}{m} \sum_{k=1}^{m} k C(k, n-1) & \text { if } 1 \leq m \leq a, n \geq 2, \\ -\frac{r}{m} \sum_{k=m-a}^{m} k C(k, n-1) & \text { if } m>a, n \geq 2, \\ 0 & \text { otherwise. }\end{cases}
$$

(b) If $a, b>0$ and $c=0$, then $H_{k}\left(\mathfrak{g}_{-}^{(J)}\right)=V_{J}\left(-k \alpha_{1}\right)^{\oplus r}$, and hence we have

$$
\begin{aligned}
\zeta(-m,-n)= & \operatorname{sdim} H\left(\mathfrak{g}_{-}^{(J)}\right)_{(-m,-n)} \\
& = \begin{cases}1 & \text { if }(m, n)=(0,0), \\
\left(\begin{array}{c}
r+n-1 \\
n
\end{array}\right) & \text { if } 0 \leq m \leq n a, n \geq 1, \\
0 & \text { otherwise. }\end{cases}
\end{aligned}
$$

Therefore we obtain

$$
\operatorname{sdim} \mathfrak{g}_{(m, n)}=C(m, n)-\sum_{\substack{d>1 \\ d \mid(m, n)}} \frac{1}{d} \operatorname{sdim} \mathfrak{g}_{\left(\frac{m}{d}, \frac{n}{d}\right)}
$$

where $C(m, n)$ are determined recursively by

$$
C(m, n)=\left\{\begin{array}{c}
\text { if } 1 \leq m \leq a, n=1, \\
-\frac{1}{m} \sum_{\substack{1 \leq l \leq n-1 \\
\sigma(l) \leq k \leq m}} k\left(\begin{array}{c}
r+n-l-1 \\
r-1
\end{array}\right) C(k, l)-\left(\begin{array}{c}
r=n-1 \\
r-1
\end{array}\right) \\
\text { if } 1 \leq m \leq n a, n \geq 2, \\
-\frac{1}{m} \sum_{\substack{1 \leq l \leq n-1 \\
\sigma(l) \leq k \leq m}} k\left(\begin{array}{c}
r+n-l-1 \\
r-1
\end{array}\right) C(k, l) \\
\text { if } m>n a, n \geq 2, \\
\text { otherwise. }
\end{array}\right.
$$


Here $\sigma(l)=\max \{1, m-a(n-l)\}$.

We present the root multiplicity tables for these generalized Kac-Moody superalgebras in Table 5.1 - Table 5.5.

EXAMPLE 5.3. Let $\mathfrak{g}$ be the rank 2 generalized Kac-Moody superalgebra considered in Example 5.2 and let $V(\Lambda)$ be the irreducible highest weight $\mathfrak{g}$-module with highest weight $\Lambda$, where $\Lambda$ is defined by $\Lambda\left(h_{i}\right)=\lambda_{i}$ $(i=0,1)$. Set

$$
W(m, n)=\operatorname{sdim} V(\Lambda)_{\Lambda-m \alpha_{0}-n \alpha_{1}} \quad\left(m, n \in \mathbb{Z}_{\geq 0}\right) .
$$

Then by the Freudenthal-type weight multiplicity formula (4.8), we obtain

$$
W(m, n)=\frac{N(m, n)}{D(m, n)},
$$

where

$$
\begin{gathered}
\begin{aligned}
N(m, n)=2 \sum_{\substack{0 \leq k \leq m \\
0 \leq l \leq n}}\left(b k \lambda_{0}+a l \lambda_{1}-b(m-k)(2 k-a l)+a(n-l)(b k+c l)\right) \\
\times C(k, l) W(m-k, n-l), \\
D(m, n)=2\left(b m \lambda_{0}+a n \lambda_{1}\right)-\left(2 b m^{2}-2 a b m n-a c n^{2}\right)+2 b m-a c n,
\end{aligned}
\end{gathered}
$$

and $C(k, l)$ are determined by the recursive formulas in Example 5.2.

In Table 5.6 - Table 5.8, we present the weight multiplicity tables for these modules over rank 2 generalized Kac-Moody superalgebra $\mathfrak{g}$.

ExAMPLE 5.4. Let $I=\{-1\} \cup\{1,2,3, \cdots\}$ be the index set and let $A=(-(i+j))_{i, j \in I}$ be the Borcherds-Cartan matrix of the Monster Lie algebra $([2])$. Consider a normalized $q$-series $F(q)=\sum_{n=-1}^{\infty} f(n) q^{n}$ such that $f(-1)=1, f(0)=0$, and $f(n) \in \mathbb{Z}$ for all $n \geq 1$. We define the charge of the matrix $A$ to be $\underline{m}=(|f(i)|: i \in I)$ and set $I^{\text {even }}=\{i \in I \mid f(i)>0\}$, $I^{\text {odd }}=\{i \in I \mid f(i)<0\}$. (We neglect those $i$ 's for which $f(i)=0$.) Then the generalized Kac-Moody superalgebra $\mathfrak{g}=\mathfrak{g}\left(A, \underline{m}, I^{\text {odd }}\right)$ is called the Monstrous Lie superalgebra associated with the normalized $q$-series $F(q)=$ $\sum_{n=-1}^{\infty} f(n) q^{n}([13])$.

We identify the simple roots $\alpha_{i}(i \in I)$ with $(1, i) \in \mathbb{Z} \times \mathbb{Z}$ and define a nondegenerate symmetric bilinear form on $\mathbb{Z} \times \mathbb{Z}$ by $((k, l) \mid(m, n))=$ $-(\operatorname{lm}+k n)$. Set $\rho=(1,0)$ so that we have $\left(\rho \mid \alpha_{i}\right)=-i=\frac{1}{2}\left(\alpha_{i} \mid \alpha_{i}\right)$ for all 
$i \in I$. We would like to apply our Peterson-type root multiplicity formula (4.6) to the Monstrous Lie superalgebra $\mathfrak{g}$.

Take $J=\{-1\}$ and consider the triangular decomposition

$$
\mathfrak{g}=\mathfrak{g}_{-}^{(J)} \oplus \mathfrak{g}_{0}^{(J)} \oplus \mathfrak{g}_{+}^{(J)}
$$

where $\mathfrak{g}_{0}^{(J)}=\left\langle e_{-1}, f_{-1}, h_{-1}\right\rangle+\mathfrak{h} \cong \operatorname{sl}(2, \mathbb{C})+\mathfrak{h}, \mathfrak{g}_{ \pm}^{(J)}=\bigoplus_{m, n=1}^{\infty} \mathfrak{g}_{( \pm m, \pm n)}$. Since $W(J)=\{1\}$, we have

$$
\begin{aligned}
& H_{0}\left(\mathfrak{g}_{-}^{(J)}\right)=\mathbb{C} \\
& H_{1}\left(\mathfrak{g}_{-}^{(J)}\right)=\bigoplus_{i=1}^{\infty} V_{J}\left(-\alpha_{i}\right)^{\oplus|f(i)|} \\
& H_{k}\left(\mathfrak{g}_{-}^{(J)}\right)=0 \quad \text { if } \quad k \geq 2
\end{aligned}
$$

where $V_{J}\left(-\alpha_{i}\right)$ is the $i$-dimensional irreducible $s l(2, \mathbb{C})$-module. Hence the denominator identity for the Lie superalgebra $\mathfrak{g}_{-}^{(J)}$ is equal to

$$
\prod_{m, n=1}^{\infty}\left(1-p^{m} q^{n}\right)^{\operatorname{sdim} \mathfrak{g}_{(m, n)}}=1-\sum_{i, j=1}^{\infty} f(i+j-1) p^{i} q^{j}
$$

where $p=E^{(-1,0)}, q=E^{(0,-1)}$. Therefore, we obtain

$$
\begin{aligned}
\operatorname{sdim} \mathfrak{g}_{(m, n)}= & f(m+n-1)-\sum_{\substack{d>1 \\
d \mid(m, n)}} \frac{1}{d} \operatorname{sdim} \mathfrak{g}_{\left(\frac{m}{d}, \frac{n}{d}\right)} \\
& +\frac{1}{n} \sum_{(m, n)=(k, l)+(s, t)} l\left(\sum_{d \mid(k, l)} \frac{1}{d} \operatorname{sdim} \mathfrak{g}_{\left(\frac{k}{d}, \frac{l}{d}\right)}\right) f(s+t-1) .
\end{aligned}
$$

In Table 5.9 and Table 5.10, we present the root multiplicity tables for the Monstrous Lie superalgebras associated with the Thompson series $T_{2 A}$ and $T_{2 B}$. 
Root multiplicity tables of $\mathfrak{g}\left(A, \underline{m}, I^{\text {odd }}\right)$ in Example 5.2

(Here each entries in the tables represent $\operatorname{sdim} \mathfrak{g}_{m \alpha_{0}+n \alpha_{1}}$.)

Table $5.1: r=1, a=2, c>0$

\begin{tabular}{|c|c|c|c|c|c|c|c|c|c|c|}
\hline$m \backslash n$ & 1 & 2 & 3 & 4 & 5 & 6 & 7 & 8 & 9 & 10 \\
\hline 1 & -1 & 1 & -1 & 1 & -1 & 1 & -1 & 1 & -1 & 1 \\
2 & -1 & 2 & -2 & 2 & -3 & 4 & -4 & 4 & -5 & 6 \\
3 & 0 & 1 & -2 & 4 & -6 & 8 & -11 & 14 & -17 & 21 \\
4 & 0 & 1 & -2 & 4 & -9 & 16 & -23 & 32 & -46 & 63 \\
5 & 0 & 0 & -1 & 4 & -10 & 21 & -38 & 63 & -98 & 145 \\
6 & 0 & 0 & 0 & 2 & -9 & 24 & -51 & 96 & -172 & 288 \\
7 & 0 & 0 & 0 & 1 & -6 & 21 & -56 & 127 & -256 & 474 \\
8 & 0 & 0 & 0 & 0 & -3 & 16 & -51 & 136 & -323 & 681 \\
9 & 0 & 0 & 0 & 0 & -1 & 8 & -38 & 127 & -348 & 835 \\
10 & 0 & 0 & 0 & 0 & 0 & 4 & -23 & 96 & -323 & 900 \\
\hline
\end{tabular}

Table $5.2: r=1, a=3, c>0$

\begin{tabular}{|c|c|c|c|c|c|c|c|c|c|c|}
\hline$m \backslash n$ & 1 & 2 & 3 & 4 & 5 & 6 & 7 & 8 & 9 & 10 \\
\hline 1 & -1 & 1 & -1 & 1 & -1 & 1 & -1 & 1 & -1 & 1 \\
2 & -1 & 2 & -2 & 2 & -3 & 4 & -4 & 4 & -5 & 6 \\
3 & -1 & 2 & -3 & 5 & -7 & 9 & -12 & 15 & -18 & 22 \\
4 & 0 & 2 & -4 & 7 & -13 & 21 & -29 & 39 & -54 & 72 \\
5 & 0 & 1 & -4 & 10 & -20 & 36 & -59 & 91 & -134 & 190 \\
6 & 0 & 1 & -3 & 10 & -27 & 57 & -104 & 176 & -288 & 449 \\
7 & 0 & 0 & -2 & 10 & -31 & 76 & -161 & 309 & -550 & 924 \\
8 & 0 & 0 & -1 & 7 & -31 & 93 & -222 & 474 & -939 & 1727 \\
9 & 0 & 0 & 0 & 5 & -27 & 96 & -274 & 666 & -1449 & 2905 \\
10 & 0 & 0 & 0 & 2 & -20 & 93 & -304 & 836 & -2039 & 4490 \\
\hline
\end{tabular}


Table $5.3: r=2, a=1, c>0$

\begin{tabular}{|c|c|c|c|c|c|c|c|c|c|c|}
\hline$m \backslash n$ & 1 & 2 & 3 & 4 & 5 & 6 & 7 & 8 & 9 & 10 \\
\hline 1 & -2 & 4 & -8 & 16 & -32 & 64 & -128 & 256 & -512 & 1024 \\
2 & 0 & 3 & -8 & 22 & -64 & 164 & -384 & 888 & -2048 & 4624 \\
3 & 0 & 0 & -2 & 16 & -64 & 212 & -640 & 1792 & -4776 & 12288 \\
4 & 0 & 0 & 0 & 3 & -32 & 164 & -640 & 2228 & -7168 & 21536 \\
5 & 0 & 0 & 0 & 0 & -6 & 64 & -384 & 1792 & -7168 & 25804 \\
6 & 0 & 0 & 0 & 0 & 0 & 11 & -128 & 888 & -4776 & 21536 \\
7 & 0 & 0 & 0 & 0 & 0 & 0 & -18 & 256 & -2048 & 12288 \\
8 & 0 & 0 & 0 & 0 & 0 & 0 & 0 & 30 & -512 & 4624 \\
9 & 0 & 0 & 0 & 0 & 0 & 0 & 0 & 0 & -56 & 1024 \\
10 & 0 & 0 & 0 & 0 & 0 & 0 & 0 & 0 & 0 & 105 \\
\hline
\end{tabular}

Table $5.4: r=2, a=2, c>0$

\begin{tabular}{|c|c|c|c|c|c|c|c|c|c|c|}
\hline$m \backslash n$ & 1 & 2 & 3 & 4 & 5 & 6 & 7 & 8 & 9 & 10 \\
\hline 1 & -2 & 4 & -8 & 16 & -32 & 64 & -128 & 256 & -512 & 1024 \\
2 & -2 & 7 & -16 & 38 & -96 & 228 & -512 & 1144 & -2560 & 5648 \\
3 & 0 & 4 & -18 & 64 & -192 & 532 & -1408 & 3584 & -8872 & 21504 \\
4 & 0 & 3 & -16 & 73 & -288 & 968 & -2944 & 8492 & -23552 & 63024 \\
5 & 0 & 0 & -8 & 64 & -326 & 1344 & -4864 & 16128 & -50176 & 148684 \\
6 & 0 & 0 & -2 & 38 & -288 & 1511 & -6528 & 25056 & -88400 & 291936 \\
7 & 0 & 0 & 0 & 16 & -192 & 1344 & -7186 & 32512 & -131072 & 485376 \\
8 & 0 & 0 & 0 & 3 & -96 & 968 & -6528 & 35386 & -165376 & 692880 \\
9 & 0 & 0 & 0 & 0 & -32 & 532 & -4864 & 32512 & -178568 & 855040 \\
10 & 0 & 0 & 0 & 0 & -6 & 228 & -2944 & 25056 & -165376 & 916949 \\
\hline
\end{tabular}

Table $5.5: r=2, a=3, c>0$

\begin{tabular}{|c|c|c|c|c|c|c|c|c|c|c|}
\hline$m \backslash n$ & 1 & 2 & 3 & 4 & 5 & 6 & 7 & 8 & 9 & 10 \\
\hline 1 & -2 & 4 & -8 & 16 & -32 & 64 & -128 & 256 & -512 & 1024 \\
2 & -2 & 7 & -16 & 38 & -96 & 228 & -512 & 1144 & -2560 & 5648 \\
3 & -2 & 8 & -26 & 80 & -224 & 596 & -1536 & 3840 & -9384 & 22528 \\
4 & 0 & 7 & -32 & 121 & -416 & 1288 & -3712 & 10284 & -27648 & 72240 \\
5 & 0 & 4 & -32 & 160 & -646 & 2304 & -7552 & 23296 & -68608 & 194764 \\
6 & 0 & 3 & -26 & 172 & -864 & 3595 & -13312 & 45656 & -147792 & 456304 \\
7 & 0 & 0 & -16 & 160 & -992 & 4864 & -20626 & 79104 & -281600 & 946176 \\
8 & 0 & 0 & -8 & 121 & -992 & 5840 & -28416 & 122274 & -480768 & 1762000 \\
9 & 0 & 0 & -2 & 80 & -864 & 6184 & -35072 & 170496 & -742448 & 2974720 \\
10 & 0 & 0 & 0 & 38 & -646 & 5840 & -38912 & 215216 & -1043968 & 4588149 \\
\hline
\end{tabular}


Weight multiplicity tables of $\mathfrak{g}\left(A, \underline{m}, I^{\text {odd }}\right)$ in Example 5.3 (Here each entries in the tables represent $W(m, n)$.)

Table $5.6: r=1, a=2, c>0, \Lambda\left(h_{0}\right)=1, \Lambda\left(h_{1}\right)=0$

\begin{tabular}{|c|c|c|c|c|c|c|c|c|c|c|c|}
\hline$m \backslash n$ & 0 & 1 & 2 & 3 & 4 & 5 & 6 & 7 & 8 & 9 & 10 \\
\hline 0 & 1 & 0 & 0 & 0 & 0 & 0 & 0 & 0 & 0 & 0 & 0 \\
1 & 1 & -1 & 1 & -1 & 1 & -1 & 1 & -1 & 1 & -1 & 1 \\
2 & 0 & -1 & 2 & -3 & 4 & -5 & 6 & -7 & 8 & -9 & 10 \\
3 & 0 & 0 & 2 & -5 & 9 & -14 & 20 & -27 & 35 & -44 & 54 \\
4 & 0 & 0 & 1 & -5 & 13 & -26 & 45 & -71 & 105 & -148 & 201 \\
5 & 0 & 0 & 0 & -3 & 13 & -35 & 75 & -140 & 238 & -378 & 570 \\
6 & 0 & 0 & 0 & -1 & 9 & -35 & 96 & -216 & 427 & -770 & 1296 \\
7 & 0 & 0 & 0 & 0 & 4 & -26 & 96 & -267 & 623 & -1288 & 2436 \\
8 & 0 & 0 & 0 & 0 & 1 & -14 & 75 & -267 & 750 & -1800 & 3858 \\
9 & 0 & 0 & 0 & 0 & 0 & -5 & 45 & -216 & 750 & -2123 & 5211 \\
10 & 0 & 0 & 0 & 0 & 0 & -1 & 20 & -140 & 623 & -2123 & 6046 \\
\hline
\end{tabular}

Table $5.7: r=1, a=2, c>0, \Lambda\left(h_{0}\right)=0, \Lambda\left(h_{1}\right)=1$

\begin{tabular}{|c|c|c|c|c|c|c|c|c|c|c|c|}
\hline$m \backslash n$ & 0 & 1 & 2 & 3 & 4 & 5 & 6 & 7 & 8 & 9 & 10 \\
\hline 0 & 1 & -1 & 1 & -1 & 1 & -1 & 1 & -1 & 1 & -1 & 1 \\
1 & 0 & -1 & 2 & -3 & 4 & -5 & 6 & -7 & 8 & -9 & 10 \\
2 & 0 & -1 & 3 & -6 & 10 & -15 & 21 & -28 & 36 & -45 & 55 \\
3 & 0 & 0 & 2 & -7 & 16 & -30 & 50 & -77 & 112 & -156 & 210 \\
4 & 0 & 0 & 1 & -6 & 19 & -45 & 90 & -161 & 266 & -414 & 615 \\
5 & 0 & 0 & 0 & -3 & 16 & -51 & 126 & -266 & 504 & -882 & 1452 \\
6 & 0 & 0 & 0 & -1 & 10 & -45 & 141 & -357 & 784 & -1554 & 2850 \\
7 & 0 & 0 & 0 & 0 & 4 & -30 & 126 & -393 & 1016 & -2304 & 4740 \\
8 & 0 & 0 & 0 & 0 & 1 & -15 & 90 & -357 & 1107 & -2907 & 6765 \\
9 & 0 & 0 & 0 & 0 & 0 & -5 & 50 & -266 & 1016 & -3139 & 8350 \\
10 & 0 & 0 & 0 & 0 & 0 & -1 & 21 & -161 & 784 & -2907 & 8953 \\
\hline
\end{tabular}


Table $5.8: r=1, a=2, c>0, \Lambda\left(h_{0}\right)=1, \Lambda\left(h_{1}\right)=1$

\begin{tabular}{|c|c|c|c|c|c|c|c|c|c|c|c|}
\hline$m \backslash n$ & 0 & 1 & 2 & 3 & 4 & 5 & 6 & 7 & 8 & 9 & 10 \\
\hline 0 & 1 & -1 & 1 & -1 & 1 & -1 & 1 & -1 & 1 & -1 & 1 \\
1 & 1 & -2 & 3 & -4 & 5 & -6 & 7 & -8 & 9 & -10 & 11 \\
2 & 0 & -2 & 5 & -9 & 14 & -20 & 27 & -35 & 44 & -54 & 65 \\
3 & 0 & -1 & 5 & -13 & 26 & -45 & 71 & -105 & 148 & -201 & 265 \\
4 & 0 & 0 & 3 & -13 & 35 & -75 & 140 & -238 & 378 & -570 & 825 \\
5 & 0 & 0 & 1 & -9 & 35 & -96 & 216 & -427 & 770 & -1296 & 2067 \\
6 & 0 & 0 & 0 & -4 & 26 & -96 & 267 & -623 & 1288 & -2436 & 4302 \\
7 & 0 & 0 & 0 & -1 & 14 & -75 & 267 & -750 & 1800 & -3858 & 7590 \\
8 & 0 & 0 & 0 & 0 & 5 & -45 & 216 & -750 & 2123 & -5211 & 11505 \\
9 & 0 & 0 & 0 & 0 & 1 & -20 & 140 & -623 & 2123 & -6046 & 15115 \\
10 & 0 & 0 & 0 & 0 & 0 & -6 & 71 & -427 & 1800 & -6046 & 17303 \\
\hline
\end{tabular}

Monstrous Lie superalgebra associated with $T_{2 A}$

(Here each entries represent $\left.\operatorname{sdimg} \mathfrak{g}_{(m, n)}\right)$

Table 5.9

\begin{tabular}{|c|l|l|l|}
\hline$m \backslash n$ & 1 & 2 & 3 \\
\hline 1 & 4372 & 96256 & 1240002 \\
2 & 96256 & 10795008 & 431529984 \\
3 & 1240002 & 431529984 & 42616961892 \\
4 & 10698752 & 10128277504 & 2125795885056 \\
5 & 74428120 & 166564106240 & 68134255043715 \\
6 & 431529984 & 2126227415040 & 1588198806411264 \\
7 & 2206741887 & 22327393665024 & 29030493318777216 \\
8 & 10117578752 & 200750502117376 & 437155796944945152 \\
9 & 42616961892 & 1588198806411264 & 5614282459787463036 \\
10 & 166564106240 & 11283779936849920 & 63071424165763399680 \\
\hline
\end{tabular}

\begin{tabular}{|c|l|l|}
\hline$m \backslash n$ & 4 & 5 \\
\hline 1 & 10698752 & 74428120 \\
2 & 10128277504 & 166564106240 \\
3 & 2125795885056 & 68134255043715 \\
4 & 200750502117376 & 11283613372743680 \\
5 & 11283613372743680 & 1040545340935546700 \\
6 & 437157922740830208 & 63071424165763399680 \\
7 & 12748902531008430080 & 2776078010473426349320 \\
8 & 296560986580914798592 & 94724982482640008642560 \\
9 & 5729955600122990051328 & 2622912698569732740150840 \\
10 & 94724993766253381386240 & 60941641644938584748902400 \\
\hline
\end{tabular}




\begin{tabular}{|c|l|l|}
\hline$m \backslash n$ & 6 & 7 \\
\hline 1 & 431529984 & 2206741887 \\
2 & 2126227415040 & 22327393665024 \\
3 & 1588198806411264 & 29030493318777216 \\
4 & 437157922740830208 & 12748902531008430080 \\
5 & 63071424165763399680 & 2776078010473426349320 \\
6 & 5729957188321796462592 & 365905674143822100234240 \\
7 & 365905674143822100234240 & 32901400981362568466623924 \\
8 & 17653726811858612022411264 & 2182844606054987051800985600 \\
9 & 676781767952182209443807232 & 112867667380626713409166646166 \\
10 & 21390391153642343366410813440 & 4734083066463091210586418544640 \\
\hline
\end{tabular}

\begin{tabular}{|c|l|}
\hline$m \backslash n$ & 8 \\
\hline 1 & 10117578752 \\
2 & 200750502117376 \\
3 & 437155796944945152 \\
4 & 296560986580914798592 \\
5 & 94724982482640008642560 \\
6 & 17653726811858612022411264 \\
7 & 2182844606054987051800985600 \\
8 & 194804003233778348867415179264 \\
9 & 13307182910011920334374580912128 \\
10 & 726344627267043576138497719009280 \\
\hline
\end{tabular}

\begin{tabular}{|c|l|}
\hline$m \backslash n$ & 9 \\
\hline 1 & 42616961892 \\
2 & 1588198806411264 \\
3 & 5614282459787463036 \\
4 & 5729955600122990051328 \\
5 & 2622912698569732740150840 \\
6 & 676781767952182209443807232 \\
7 & 112867667380626713409166646166 \\
8 & 13307182910011920334374580912128 \\
9 & 1180772370567563904378531394829748 \\
10 & 82539410782781631041963275207495680 \\
\hline
\end{tabular}




\begin{tabular}{|c|l|}
\hline$m \backslash n$ & 10 \\
\hline 1 & 166564106240 \\
2 & 11283779936849920 \\
3 & 63071424165763399680 \\
4 & 94724993766253381386240 \\
5 & 60941641644938584748902400 \\
6 & 21390391153642343366410813440 \\
7 & 4734083066463091210586418544640 \\
8 & 726344627267043576138497719009280 \\
9 & 82539410782781631041963275207495680 \\
10 & 7290799226637980819592664955451443200 \\
\hline
\end{tabular}

Monstrous Lie superalgebra associated with $T_{2 B}$

(Here each entries in the tables represent $\operatorname{sdimg}(m, n)$ )

Table 5.10

\begin{tabular}{|c|l|l|l|l|}
\hline$m \backslash n$ & 1 & 2 & 3 & 4 \\
\hline 1 & 276 & -2048 & 11202 & -49152 \\
2 & -2048 & 49152 & -614400 & 5373952 \\
3 & 11202 & -614400 & 14478180 & -216072192 \\
4 & -49152 & 5373952 & -216072192 & 5061476352 \\
5 & 184024 & -37122048 & 2390434947 & -83300614144 \\
6 & -614400 & 216072192 & -21301241856 & 1063005978624 \\
7 & 1881471 & -1102430208 & 160791890304 & -11164248047616 \\
8 & -5373952 & 5061476352 & -1063005978624 & 100372723007488 \\
9 & 14478180 & -21301241856 & 6300794030460 & -794110053826560 \\
10 & -37122048 & 83300614144 & -34065932304384 & 5641848336678912 \\
\hline
\end{tabular}

\begin{tabular}{|c|l|l|}
\hline$m \backslash n$ & 5 & 6 \\
\hline 1 & 184024 & -614400 \\
2 & -37122048 & 216072192 \\
3 & 2390434947 & -21301241856 \\
4 & -83300614144 & 1063005978624 \\
5 & 1945403602764 & -34065932304384 \\
6 & -34065932304384 & 794110053826560 \\
7 & 478625723149576 & -14515166263443456 \\
8 & -5641848336678912 & 218578429975461888 \\
9 & 57567784186189368 & -2807138079496716288 \\
10 & -520271697765971968 & 31535729115847852032 \\
\hline
\end{tabular}




\begin{tabular}{|c|l|l|}
\hline$m \backslash n$ & 7 & 8 \\
\hline 1 & 1881471 & -5373952 \\
2 & -1102430208 & 5061476352 \\
3 & 160791890304 & -1063005978624 \\
4 & -11164248047616 & 100372723007488 \\
5 & 478625723149576 & -5641848336678912 \\
6 & -14515166263443456 & 218578429975461888 \\
7 & 337945040343588276 & -6374456847628238848 \\
8 & -6374456847628238848 & 148280443106626633728 \\
9 & 101150679669913197462 & -2864978197116521938944 \\
10 & -1388038765923851599872 & 47362494062244172660736 \\
\hline
\end{tabular}

\begin{tabular}{|c|l|l|}
\hline$m \backslash n$ & 9 & 10 \\
\hline 1 & 14478180 & -37122048 \\
2 & -21301241856 & 83300614144 \\
3 & 6300794030460 & -34065932304384 \\
4 & -794110053826560 & 5641848336678912 \\
5 & 57567784186189368 & -520271697765971968 \\
6 & -2807138079496716288 & 31535729115847852032 \\
7 & 101150679669913197462 & -1388038765923851599872 \\
8 & -2864978197116521938944 & 47362494062244172660736 \\
9 & 66600077798590855556532 & -1311456320500974276980736 \\
10 & -1311456320500974276980736 & 304708210826051412574371 \\
\hline
\end{tabular}

\section{REFERENCES}

[1] R. E. Borcherds, Generalized Kac-Moody algebras, J. Algebra, 115 (1988), 501-512.

[2] L Monstrous moonshine and monstrous Lie superalgebras, Invent. Math., 109 (1992), 405-444.

[3], Automorphic forms on $O_{s+2,2}(\mathbb{R})$ and infinite products, Invent. Math., 120 (1995), 161-213.

[4] H. Cartan and S. Eilenberg, Homological algebra, Princeton Mathematics Series, Princeton University, 1956.

[5] J. H. Conway and S. Norton, Monstrous moonshine, Bull. Lond. Math. Soc., 11 (1979), 308-339.

[6] D. B. Fuks, Cohomology of infinite dimensional Lie algebras, Consultant Bureau, New York, 1986.

[7] Y.-J. Im, Defining relations of generalized Kac-Moody superalgebras, M. S. thesis, Seoul National University (1998).

[8] V. G. Kac, Infinite-dimensional Lie algebras and Dedekind's $\eta$-function, Funct. Anal. Appl., 8 (1974), 68-70.

[9] L Lie superalgebras, Adv. in Math., 26 (1977), 8-96. 
[10] - Infinite dimensional algebras, Dedekind's $\eta$-function, classical Möbius function and the very strange formula, Adv. in Math., 30 (1978), 85-136.

[11] _ Infinite Dimensional Lie Algebras, Cambridge Univ. Press, 3rd ed., 1990.

[12] S.-J. Kang, Generalized Kac-Moody algebras and the modular function j, Math. Ann., 298 (1994), 373-384.

[13] _ Graded Lie superalgebras and the superdimension formula, J. Algebra, 204 (1998), 597-655.

[14] S.-J. Kang and M.-H. Kim, Dimension formula for graded Lie algebras and its applications, Trans. Amer. Math. Soc., 351 (1999), 4281-4336.

[15] S.-J. Kang and J.-H. Kwon, Graded Lie superalgebras, supertrace formula, and orbit Lie superalgebra, Proc. London Math. Soc., 81 (1998), 675-724.

[16] S.-J. Kang and Y.-T. Oh, Peterson-type supertrace formula for graded Lie superalgebras and super-replicable functions, in preparation.

[17] J.-K. Koo and Y.-T Oh, Freudenthal-type multiplicity formulas for generalized Kac-Moody superalgebras, Comm. Algebra, 29(1) (2001), 225-244.

[18] L.-S. Liu, Kostant's formula for Kac-Moody Lie algebras, J. Algebra, 149 (1992), $155-178$.

[19] I. G. Macdonald, Affine root systems and Dedekind's $\eta$-function, Invent. Math., 15 (1972), 91-143.

[20] I. G. Macdonald, Symmetric Functions and Hall Polynomials, Oxford Univ. Press, England, 2nd ed., 1995.

[21] M. Miyamoto, A generalization of Borcherds algebra and denominator formula, J. Algebra, 180 (1996), 631-651.

[22] D. H. Peterson, Freudenthal-type formulas for root and weight multiplicities, Preprint (1983), unpublished.

[23] U. Ray, A character formula for generalized Kac-Moody superalgebras, J. Algebra, 177 (1995), 154-163.

Seok-Jin Kang

Korea Institute for Advanced Study

20\%-43 Cheongryangri-dong, Dongdaemun-gu

Seoul 130-012, Korea

sjkang@kias.re.kr

Jae-Hoon Kwon

Korea Institute for Advanced Study

207-43 Cheongryangri-dong, Dongdaemun-gu

Seoul 130-012, Korea

jhkwon@math.snu.ac.kr

Young-Tak Oh

Department of Mathematics

Seoul National University

Seoul 151-742, Korea

ohyt@math.snu.ac.kr 